\title{
A Feed-Forward Subnetwork Emerging from Integrated TOR- and cAMP/PKA-Signaling Architecture Reinforces Magnaporthe oryzae Appressorium Morphogenesis
}

\author{
Guangchao Sun, Xiaobo Qi, and Richard A. Wilson ${ }^{+}$ \\ Department of Plant Pathology, University of Nebraska-Lincoln, Lincoln, NE, U.S.A.
}

Accepted 10 November 2018.

\begin{abstract}
Appressoria are important mediators of plant-microbe interactions. In the devastating rice blast pathogen Magnaporthe oryzae, appressorial morphogenesis from germ tube tips requires activated cAMP/PKA signaling and inactivated TOR signaling $\left(T_{O} R_{\text {off }}\right)$. TOR off $_{\text {temporarily arrests }}$ G2 at a metabolic checkpoint during the single round of mitosis that occurs following germination. G2 arrest induces autophagy and appressorium formation concomitantly, allowing reprogression of the cell cycle to G1/G0 quiescence and a single appressorial nucleus. Inappropriate TOR activation abrogates G2 arrest and inhibits cAMP/PKA signaling downstream of cPKA. This results in multiple rounds of germ tube mitosis and the loss of autophagy and appressoria formation. How cAMP/PKA signaling connects to cell cycle progression and autophagy is not known. To address this, we interrogated TOR and cAMP/PKA pathways using signaling mutants, different surface properties, and specific cell cycle inhibitors and discovered a feed-forward subnetwork arising from TOR- and cAMP/PKA-signaling integration. This adenylate cyclase-cAMP-TOR-adenylate cyclase subnetwork reinforces cAMP/PKA-dependent appressorium formation under favorable environmental conditions. Under unfavorable conditions, the subnetwork collapses, resulting in reversible cell cycle-mediated germ tube growth regardless of external nutrient status. Collectively, this work provides new molecular insights on germ tube morphogenetic decision-making in response to static and dynamic environmental conditions.
\end{abstract}

Keywords: appressoria, autophagy, cAMP/PKA, cell cycle, Magnaporthe oryzae, morphogenesis, Pyricularia oryzae, rice blast, TOR

Eukaryotic filamentous plant pathogens have the potential to undermine global food security (Fisher et al. 2012), and yet, affordable, sustainable, and deployable solutions are mostly not realized. Many fungi and oomycetes use specialized infection

\section{${ }^{\dagger}$ Corresponding author: R. A. Wilson: rwilson10@unl.edu}

Funding: This work was supported by a National Science Foundation Directorate for Biological Sciences grant (IOS-1557943) to R. A. Wilson. The China Scholarship Council supported G. Sun and X. Qi.

*The $\boldsymbol{e}$-Xtra logo stands for "electronic extra" and indicates that two supplementary figures and three supplementary tables are published online.

(c) 2019 The American Phytopathological Society structures called appressoria to rupture host plant surfaces and colonize underlying tissue (Emmett and Parbery 1975). Understanding the molecular underpinnings of appressoria development might reveal novel targets for disease management strategies or open new avenues for plant pathology research. The devastating rice blast fungus Magnaporthe oryzae (syn. Pyricularia oryzae; which is also an emerging threat to wheat) develops appressoria under nutrient-free conditions on hydrophobic surfaces (DiPietro and Talbot 2017; Wilson and Talbot 2009). Three-celled $M$. oryzae asexual spores (conidia) are transmitted to rice leaves by rain or wind where they adhere to the waxy cuticle and germinate. By 4 to $6 \mathrm{~h}$ postinoculation (hpi), the polarized germ tube hooks and starts to swell isotropically. The germ tube tip swelling becomes a nascent appressorium that, following a single round of mitosis around 8 hpi and subsequent autophagic cell death of the conidium (Veneault-Fourrey et al. 2006), matures by 24 hpi into an infection-competent, dome-shaped cell. Melanization of the appressorium cell wall and the generation of massive hydrostatic turgor from glycerol facilitate the penetration of the rice cuticle by a peg structure emerging from the base of the appressorium (Dagdas et al. 2012; Fernandez and Wilson 2012).

Appressorium formation requires three signaling pathways conserved in eukaryotes. cAMP/PKA signaling is required for initiating appressorium morphogenesis at germ tube tips and is activated following the production of cAMP by the adenylate cyclase Mac1 in response to inductive hydrophobic surfaces (Wilson and Talbot 2009). The mitogen-activated protein kinase pathway mediated by Pmk1 acts downstream of cAMP/PKA signaling (Xu and Hamer 1996). Recently, we determined that the cell-intrinsic TOR nutrient-sensing pathway acts downstream of cPKA but upstream of Pmk1 and controls cell cycle progression, autophagy, and appressorium formation (Marroquin-Guzman and Wilson 2015). TOR (target of rapamycin) is a conserved signaling pathway in eukaryotes mediated by Tor kinase that is activated $\left(\mathrm{TOR}_{\mathrm{on}}\right)$ in response to available nutrient in order to promote cell growth and development (Loewith and Hall 2011). Starvation conditions inactivate TOR ( $\left.\mathrm{TOR}_{\text {off }}\right)$, leading to growth suppression and autophagy (Hallet et al. 2014). Rapamycin (Rap) is the specific TOR inhibitor (Crespo and Hall 2002). In M. oryzae, appressorium morphogenesis involves a cell fate switch mediated by a TOR-dependent metabolic checkpoint at G2 (Marroquin-Guzman et al. 2017b). Specifically, TOR $_{\text {off }}$ during spore germination on inductive nutrient-free, hydrophobic surfaces arrests mitosis in the G2 phase (MarroquinGuzman et al. 2017b). G2 arrest concomitantly induces both appressorium formation and autophagy. Autophagy liberates 
nutrients, likely including glutamine, which activates TOR to restart cell cycle progression through mitosis to the $\mathrm{TOR}_{\mathrm{off}^{-}}$ dependent G1/G0 arrest of the appressorial nucleus (Marroquin-Guzman et al. 2017b). Following host infection, TOR $_{\text {on }}$ is activated in response to a metabolic switch to glucose metabolism and is required for nuclear proliferation during growth in rice cells (Fernandez et al. 2014). TOR activity status during appressorium formation is mediated by glucose signaling through $A B L 1$ (Marroquin-Guzman et al. $2017 b$ ). In the absence of exogenous glucose, the $A B L 1$ gene product inactivates TOR to mediate TOR $_{\text {off }}$ engagement of the G2 metabolic checkpoint and subsequent appressorial differentiation. Exogenous glucose treatment of germinating spores prevents the $A B L 1$ gene product from inactivating TOR (and, thus, phenocopies the $\Delta a b l l$ mutation) (MarroquinGuzman et al. 2017b). Inappropriate $\mathrm{TOR}_{\text {on }}$ during spore germination due to exogenous glucose treatment or the loss of $A B L 1$ inhibits cAMP/PKA signaling, abrogates G2 arrest, leading to multiple rounds of accelerated mitosis in germ tubes, and abolishes autophagy and appressorium formation (Marroquin-Guzman and Wilson 2015; Marroquin-Guzman et al. 2017b). In addition to glucose-signaling, exquisite control of glutamine metabolism by the GATA-factor Asd4 is critical during appressoria morphogenesis in order to prevent glutamine, a TOR agonist, from reaching intracellular concentrations sufficient to activate TOR prematurely and inhibit cAMP/PKA signaling (Marroquin-Guzman and Wilson 2015).

Despite recent advances in understanding the role of TOR- and cAMP/PKA signaling in appressorial morphogenesis, mechanistic questions regarding the interplay of environment and development remain. In particular, it is not known how one or both cell cycle progression and autophagy are affected by noninductive nutrient-free, hydrophilic surfaces (such as glass slides) that support spore germination but not appressorium formation (Marroquin-Guzman and Wilson 2015; Wilson and Talbot 2009). Determining whether spore germination on nutrientfree hydrophilic surfaces is accompanied by multiple rounds of mitosis and the loss of autophagy (as on glucoserich hydrophobic surfaces) or results in limited mitosis and the induction of autophagy (due to the external starvation conditions) would provide us with a more detailed account of the rules governing infection-related development. In this study, we compared cell cycle control and autophagy following spore germination in TOR- and cAMP/PKApathway mutants under hydrophobic and hydrophilic surface conditions and following the application of, singly or in combination, glucose, cAMP, Rap, or specific cell cycle inhibitors. In doing so, we uncovered evidence for more extensive wiring between TOR- and cAMP/PKA-signaling pathways than previously anticipated. From this integrated pathway architecture, we elaborate a feed-forward subnetwork reinforcing appressorium formation under favorable, inductive conditions and demonstrate how its collapse facilitates similar and reversible morphogenetic outcomes to different noninductive conditions and treatments. We conclude that the integration of nutrient- and surfacesensing pathways during spore germination constitutes a rapid and dynamic morphogenetic decision-making strategy highly responsive to static and changing environmental conditions. Collectively, this work enhances our fundamental understanding of cell cycle regulation, autophagy control and appressorial formation in a devastating plant pathogen and also points to a tightly controlled, but hitherto unrecognized, growth-mediated escape mechanism for germinating spores on unfavorable surfaces.

\section{RESULTS AND DISCUSSION}

Nutrient-free hydrophilic surfaces induce multiple rounds of germ tube mitosis and septa, and inhibit conidial nuclei degradation, in germinating spores.

We first sought to determine how spore germination on noninductive nutrient-free, hydrophilic surfaces affected autophagy and mitosis. Our hypothesis - that the lack of nutrients on hydrophilic surfaces would not support multiple rounds of mitosis in germ tubes and might instead stimulate autophagy and the destruction of conidial nuclei — was proven to be false when, using our otherwise wild-type (WT) Guy11 strain expressing histone $\mathrm{H} 1$ fused to red fluorescent protein (WT H1: RFP) (Marroquin-Guzman et al. 2017b), we discovered that spore germination on nutrient-free hydrophilic surfaces was accompanied by both multiple rounds of mitosis in extended germ tubes and the loss of autophagy-associated conidial nuclei degradation (Fig. 1A). Moreover, whereas on hydrophobic surfaces the single round of mitosis occurred by $8 \mathrm{hpi}$ (in our study) and autophagic degradation of conidial nuclei was complete by 24 hpi (Fig. 1A and B), mitosis in germinating spores on hydrophilic surfaces was accelerated and occurred every $4 \mathrm{~h}$ for at least the first two rounds of mitosis (Fig. 1A and B). This indicated that, as on hydrophobic surfaces following glucose treatment (Marroquin-Guzman et al. 2017b), G2 arrest was abrogated on hydrophilic surfaces, resulting in multiple, accelerated rounds of mitosis and the loss of autophagyassociated conidial nuclei degradation.

Compared with hydrophobic surfaces, germ tubes elaborating on hydrophilic surfaces also underwent multiple rounds of septation corresponding with the observed extra rounds of mitosis (Fig. 1C). Multiple septation events also were observed in spores germinating on glucose-rich hydrophobic surfaces (Marroquin-Guzman et al. 2017b).

Together, these results revealed unanticipated similarities between spore germination on noninductive nutrient-free, hydrophilic surfaces compared with spore germination on glucose-rich, hydrophobic surfaces. This suggested that in order to mediate similar developmental outcomes in response to different unfavorable environmental conditions, interactions between glucose-sensing and surface-sensing pathways might occur, thus warranting further investigation.

\section{Hydrophilic surfaces abolish autophagy induction during spore germination.}

As previously observed for glucose-treated spores germinating on hydrophobic surfaces (Marroquin-Guzman et al. 2017b), germination on hydrophilic surfaces did not result in conidial nuclear degradation (Fig. 1A). This might indicate autophagy is not induced because autophagic machinery is required for degradation of nonessential nuclear portions in yeast (Krick et al. 2008), while conidial nuclear degradation in M. oryzae following appressoria development on hydrophobic surfaces requires at least the macroautophagy components Atg 1 and Atg4 (He et al. 2012). Alternatively, it is conceivable that when spores germinate on nutrient-free hydrophilic surfaces, autophagy is induced but uncoupled from conidial nuclear degradation, potentially as a means to fuel mitosis and germ tube growth in the absence of exogenous glucose. To assess these alternative scenarios, we addressed the status and role of autophagy during spore germination on hydrophilic surfaces by staining autophagosomes and autophagic vacuoles with the acidotropic dye monodansylcadaverine (MDC) (Klionsky et al. 2016) and by generating mutant strains that were either expressing the autophagosome tethered autophagy component Atg8 fused to green fluorescent protein (GFP) at the $\mathrm{N}$ terminus (Kershaw and Talbot 2009) or were disrupted for ATG8 gene 
function. Figure 2A shows that compared with hydrophobic surfaces where MDC-labeled autophagosomal structures were localized to the appressorium by 24 hpi and were no longer evident in germ tubes, WT germ tubes on hydrophilic surfaces carried MDC-stained compartments at the tip and close to the spore, suggesting autophagy was either misregulated and/or autophagic structures were mislocalized in germ tubes on hydrophilic surfaces. This pattern of distribution of MDC-labeled autophagosomal structures in germ tubes on hydrophilic surfaces was similar to those observed in germ tubes of glucosetreated spores on hydrophobic surfaces (Marroquin-Guzman et al. 2017b), a condition under which we would not expect autophagy to be active. Furthermore, although MDC labeling is a marker for autophagic vacuoles and is upregulated during autophagy, MDC dyes are not specific markers for early autophagosomes and can also label vacuoles and degradation products (Klionsky et al. 2016). Indeed, MDC labeling can occur in autophagy-deficient mutants and in the absence of autophagy induction (Klionsky et al. 2016). Therefore, MDC labeling should be combined with other indicators of autophagy. Thus, we next compared MDC-labeling distribution in WT with distribution in the $\Delta a t g 8$ mutant strain during spore germination on hydrophobic and hydrophilic surfaces. $\triangle a t g 8$ forms nonfunctional appressoria (Veneault-Fourrey et al. 2006), and unlike WT, $\Delta a t g 8$ appressoria did not accumulate
MDC-labeled compartments (Fig. 2B), as would be expected if MDC was specifically labeling autophagosomal structures and autophagic vacuoles. However, by 24 hpi on hydrophilic surfaces, $\Delta a t g 8$ germ tubes did have a number of MDC-labeled compartments like WT, suggesting these are not autophagosomal structures. These results indicate MDC did not specifically label autophagosomal structures on hydrophilic surfaces and suggest autophagy was not induced during spore germination under these unfavorable conditions.

Further evidence that autophagy is not induced on hydrophilic surfaces is shown in Figure 2C. By 24 hpi on hydrophobic surfaces, GFP:Atg8 was observed localizing in appressoria as numerous puncta, as previously reported (Kershaw and Talbot 2009), but on hydrophilic surfaces the number of GFP:Atg8 puncta observed in germ tubes at $24 \mathrm{hpi}$ was greatly diminished and barely visible, suggesting GFP: Atg8 was not present in high levels. However, when we added the autophagy stimulator amiodarone chloride (AM) (Fleming et al. 2011) to germinating spores on hydrophilic surfaces, the number of GFP:Atg8 puncta in germ tubes significantly increased compared with the untreated control (Fig. 2C and D), and the intensity of GFP:Atg8 fluorescence also increased, which together suggest that while some basal autophagy might have occurred under untreated hydrophilic conditions, autophagy was not induced. Furthermore, as shown in Figure 2E
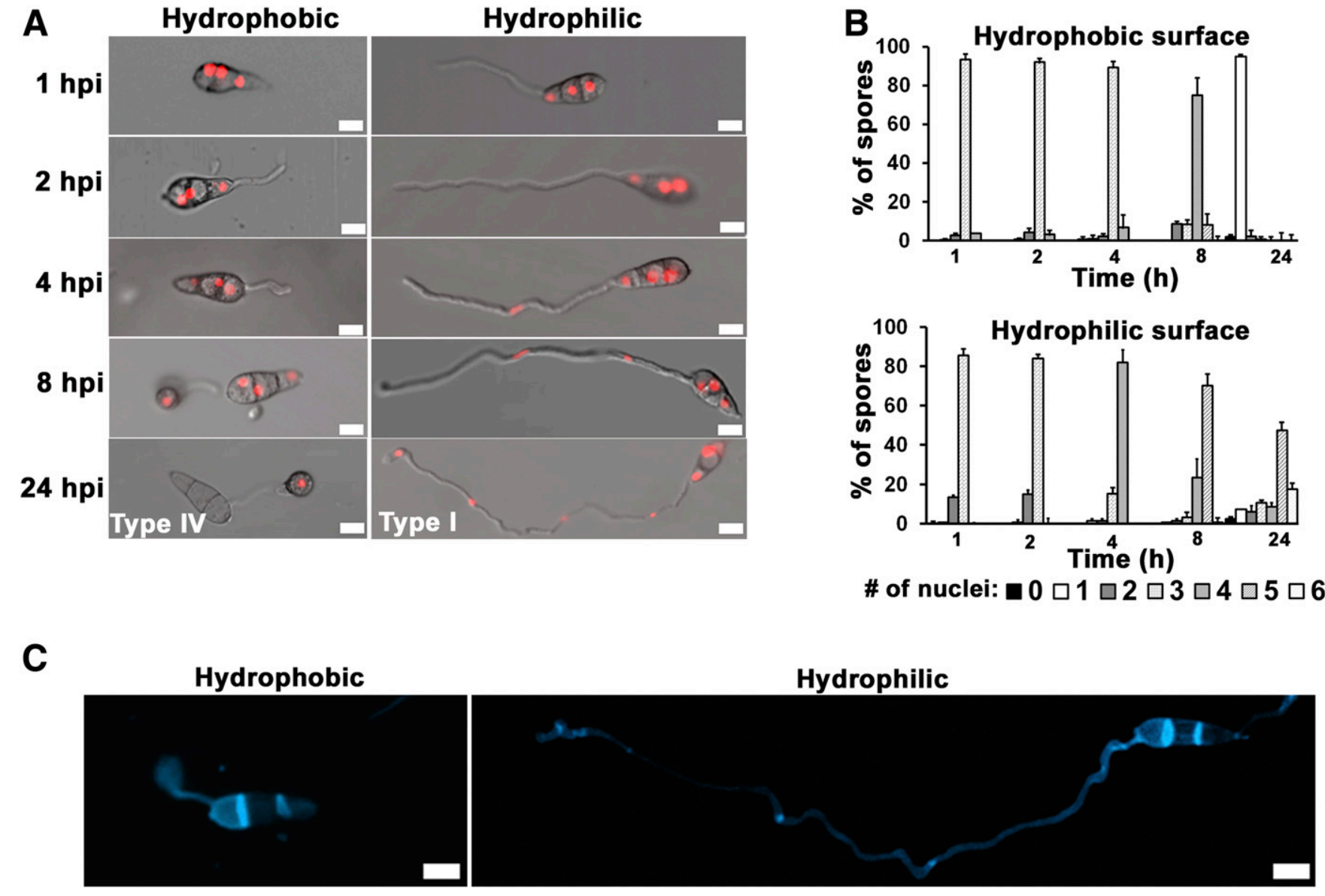

Fig. 1. Germinating spores on nutrient-free hydrophilic surfaces underwent multiple rounds of mitosis and septation accompanied by the loss of conidial nuclear degradation. A, Micrographs showing the time course of nuclear dynamics during spore germination of the wild-type (WT) Guy11 strain, expressing histone $\mathrm{H} 1$ fused to red fluorescent protein (WT H1:RFP), on hydrophobic (left panel) and hydrophilic (right panel) surfaces using laser scanning confocal microscopy. On nutrient-free hydrophobic surfaces, appressorium formation involved one round of mitosis followed by autophagic cell death of conidial nuclei. Multiple rounds of mitosis and loss of autophagic nuclear degradation were observed on hydrophilic surfaces (quantified as follows). B, Bars are the mean number of nuclei carried by 100 germinating WT H1:RFP spores on three hydrophilic slides, repeated in triplicate, at the times indicated. Error bars indicate standard deviation (SD). C, Septation events visualized by laser scanning confocal microscopy following 10 min of Calcofluor white staining of germinated WT H1:RFP spores on hydrophobic and hydrophilic surfaces at $24 \mathrm{~h}$ postinoculation. A and C, Scale bars are $10 \mu \mathrm{m}$. 
and F, AM-dependent autophagy induction on hydrophilic surfaces resulted in nuclear degradation, indicating autophagy was not uncoupled from nuclear degradation on hydrophilic surfaces but was instead not induced under these conditions. It is noteworthy that autophagy stimulation by AM was not sufficient to induce appressoria. However, when treatments with the specific TOR kinase inhibitor Rap or $10 \mathrm{mM}$ monobutyryl cyclic adenosine monophosphate (cAMP, the activator of
cAMP/PKA signaling) were applied to remediate appressorium formation on hydrophilic surfaces (Marroquin-Guzman and Wilson 2015), these treatments also restored GFP:Atg8 puncta to the appressorium (Fig. 2C).

SYTO green-fluorescent nucleic acid staining showed that $\Delta a t g 8$ conidial nuclei were not degraded by 24 hpi on hydrophobic surfaces (Fig. 2G), thus providing more evidence that macroautophagy is required for conidial nuclear degeneration
A

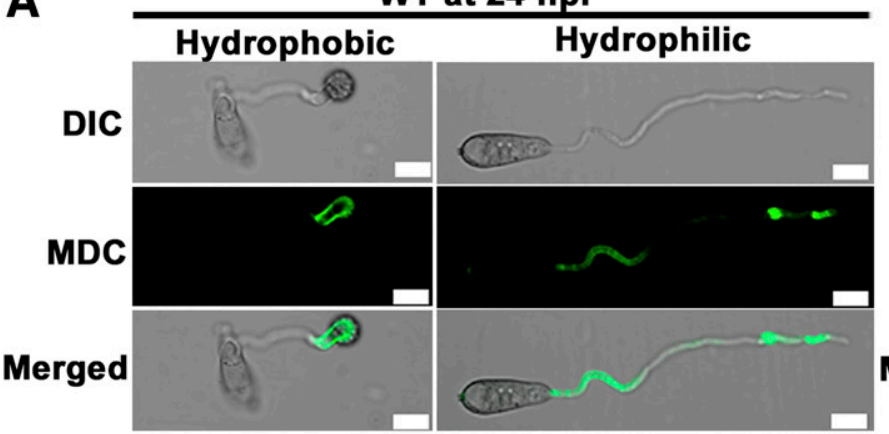

C

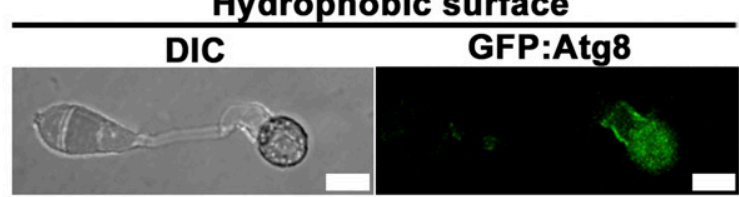

Hydrophilic surface

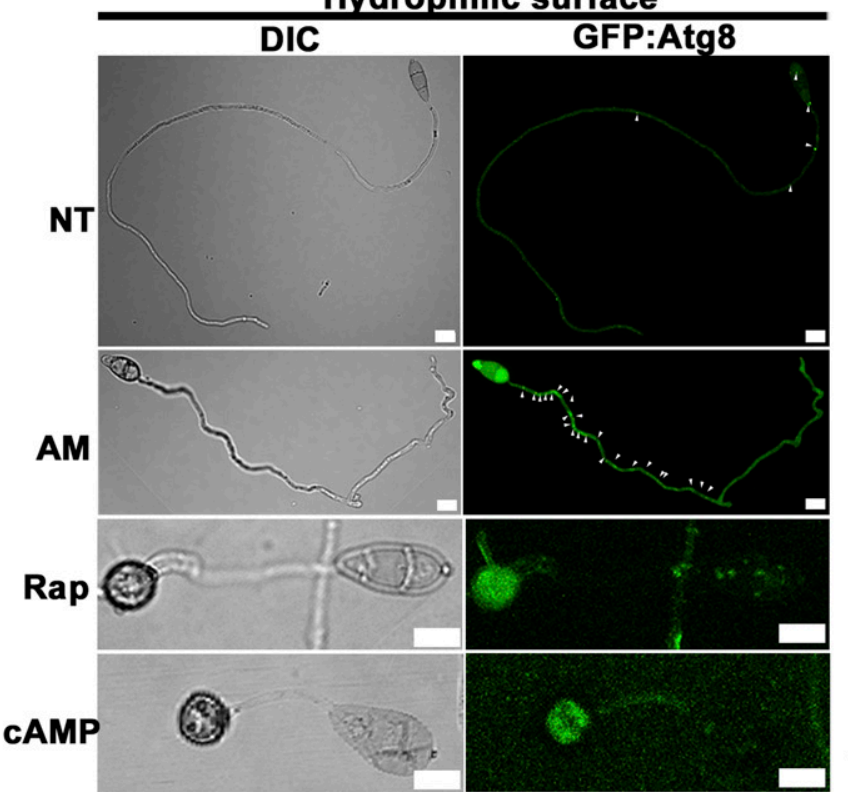

B

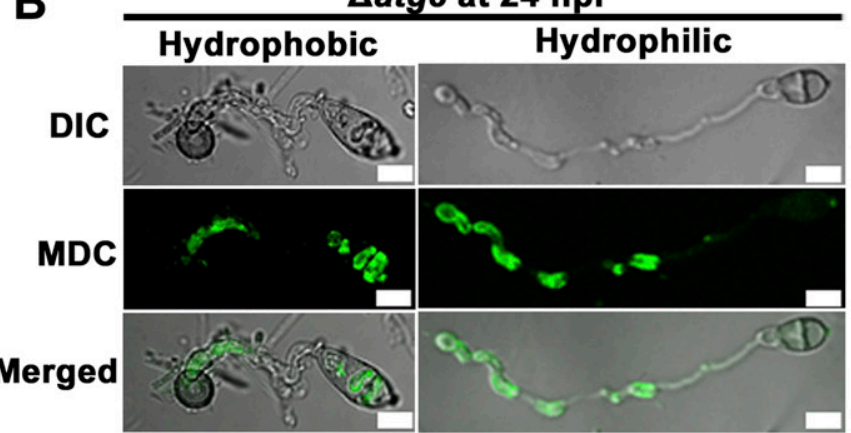

D

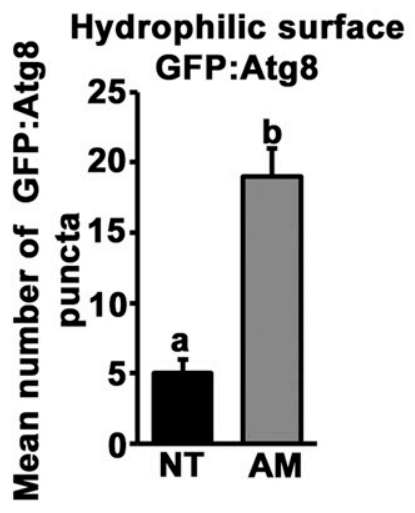

$\mathbf{F}$

Hydrophilic surface WT H1:RFP

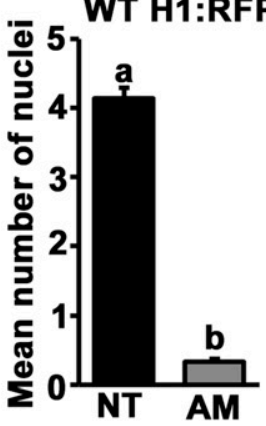

E

WT H1:RFP viewed at $24 \mathrm{hpi}$

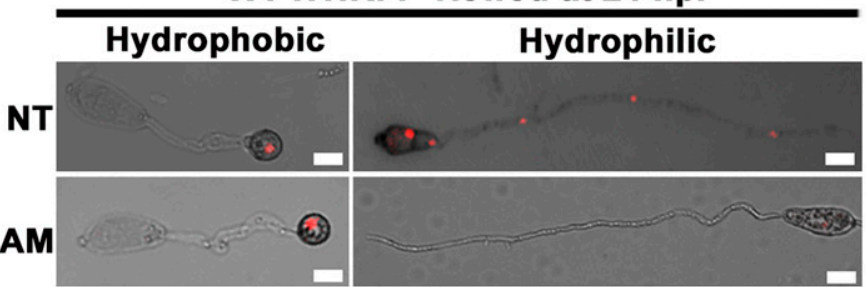

$\Delta$ atg8 at $24 \mathrm{hpi}$

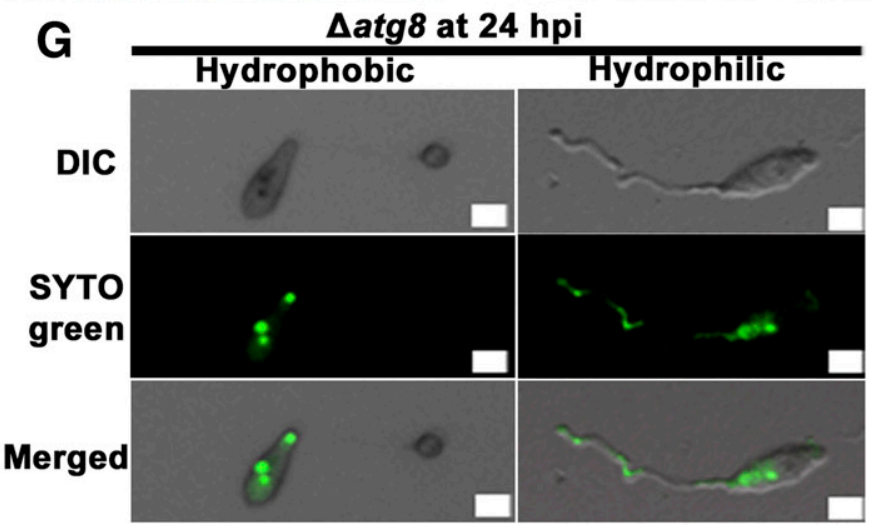


on hydrophobic surfaces. In addition, SYTO green staining showed that by $24 \mathrm{hpi}$, germinating $\Delta a t g 8$ spores under hydrophilic conditions underwent multiple rounds of mitosis and exhibited germ tube growth like WT. These results preclude a role for autophagy in germ tube growth and mitosis, although other pathways and processes are likely necessary for liberating nutrients from conidial stores in order to facilitate growth.

We conclude that autophagy induction results in conidial nuclei degradation and is required but alone is not sufficient for appressorial development. This is consistent with our previous and converse observation that inhibiting autophagy during spore germination on hydrophobic surfaces prevented conidial nuclear degeneration but not appressorial cell development (Marroquin-Guzman et al. 2017b). We also demonstrate here that autophagy is not induced on hydrophilic surfaces and is not required for the observed additional rounds of mitosis and germ tube growth.

\section{WT spores on hydrophilic surfaces \\ display restricted germ tube morphotype plasticity.}

The results shown in Figures 1 and 2, obtained under nutrient-free, hydrophilic conditions, are striking in that they resemble the effect of exogenous glucose treatment on germinating spores under hydrophobic conditions (MarroquinGuzman et al. 2017b), with both treatments resulting in multiple rounds of mitosis and the loss of autophagy induction and appressorium formation. However, unlike glucose treatment on hydrophobic surfaces, nutrient-free hydrophilic conditions did not promote germ tube morphotype plasticity. Germ tube morphotype plasticity following glucose treatment on hydrophobic surfaces was attributable to the loss of TOR-dependent cell cycle tuning, resulting in individual germinating WT spores that, by $24 \mathrm{hpi}$, produced one of four stochastic morphotypes (Marroquin-Guzman et al. 2017b): the type I morphotype is produced by about $22 \%$ of germinating spores on glucose-rich hydrophobic surfaces and presents as an extended germ tube with no appressorium; type II (formed by 5\% of germinating spores) presents as an extended germ tube with an appressorium; type III (formed by $45 \%$ of germinating spores) is a short germ tube with swollen tip and no appressorium; type IV (formed by $28 \%$ of germinating spores) is a short germ tube with an appressorium that is indistinguishable from appressoria formed under nutrient-free hydrophobic conditions (Fig. 3A, left panel; Supplementary Fig. S1; Supplementary Table S1). In contrast, as shown in Figures 1A and 3A (right panel) only the type I morphotype was produced by germinating WT spores on nutrient-free hydrophilic surfaces. This predominance of the type I morphotype under nutrient-free, hydrophilic conditions was intriguing because, not only did it show major deviation from the morphogenetic program instigated by glucose-rich hydrophobic conditions, it also divorced morphotype plasticity from mitosis and autophagy by showing that multiple rounds of mitosis and the loss of autophagy did not by themselves result in morphotype plasticity.

The type I morphotype was also the only observed morphotype when glucose was added to germinating WT spores on hydrophilic surfaces (Fig. 3A, right panel). However, the type IV morphotype was predominantly observed when 100 to $200 \mathrm{nM}$ Rap was added to WT spores on hydrophilic surfaces (Fig. 3A, right panel). As on hydrophobic surfaces (Fig. 3A, right panel), glucose did not override TOR inactivation by Rap on hydrophilic surfaces (Fig. 3A, right panel). These results suggest that on both hydrophilic and hydrophobic surfaces, TOR acts downstream of the glucose signal, but on hydrophilic surfaces the glucose signal does not override the surface signal to produce morphotypes.

Taken together, these results demonstrate that WT spores germinating on nutrient-free hydrophilic surfaces resembled germinating spores on glucose-rich hydrophobic surfaces and responded similarly to Rap, but morphotype plasticity was lost.

\section{Hydrophilic surfaces abolish morphotype plasticity in germinating $\Delta a b l 1$ spores.}

On hydrophobic surfaces, glucose regulates mitosis during germination via $A B L 1$ control of TOR (Marroquin-Guzman et al. 2017b). Germinating $\Delta a b l 1$ spores on nutrient-free hydrophobic surfaces form a range of morphotypes (type I to type IV) by 24 hpi (Fig. 3B, left panel) and resemble glucose-treated WT spores on hydrophobic surfaces, as previously described (Marroquin-Guzman et al. 2017b). We assessed morphotype diversity of germinating $\Delta a b l 1$ spores on nutrient-free hydrophilic surfaces and found that, like WT, $\Delta a b l 1$ spores only produced type I morphotypes, even in the presence of exogenous glucose (Fig. 3B, right panel). Rap treatment of $\Delta a b l 1$ spores generated mostly type IV morphotypes on hydrophilic surfaces, with or without exogenous glucose, although like WT, some germinating spores produced type III morphotypes (i.e., short germ tube with fattened tip but no appressoria) following Rap treatment (Fig. 3B, right panel).

The nuclear dynamics of the type I $\triangle a b l 1 \mathrm{H} 1$ :RFP morphotype formed on hydrophilic surfaces was indistinguishable from the $32 \%$ of germinating $\triangle a b l 1 \mathrm{H} 1$ :RFP spores that formed the type I morphotype on hydrophobic surfaces (Fig. 3C). Compared with WT on hydrophobic surfaces (Fig. 1B), the rate and number of rounds of mitosis increased for germinating $\Delta a b l 1 \mathrm{H} 1:$ RFP spores on hydrophilic surfaces, in a manner that was similar to that observed for $\triangle a b l 1 \mathrm{H} 1$ :RFP on hydrophobic surfaces, despite morphotype plasticity being abolished on hydrophilic surfaces (Fig. 3D). As on hydrophobic surfaces (Marroquin-Guzman et al. 2017b), appressorium formation by germinating $\triangle a b l 1 \mathrm{H} 1$ :RFP spores was not restored on hydrophilic surfaces by treatment with $10 \mathrm{mM}$ cAMP, indicating the glucose- $A B L 1$-TOR signaling axis remained downstream of cPKA on hydrophilic surfaces (Fig. 3E). Thus, with the exception of restricted morphotype plasticity, germinating $\Delta a b l 1$

Fig. 2. Hydrophilic surfaces impacting autophagy during spore germination. A and $\mathbf{B}$, Monodansylcadaverine (MDC)-labeled compartments in germinating wild type (WT) (A) and $\Delta a t g 8$ (B) spores, on hydrophobic and hydrophilic surfaces, viewed at 24 h postinoculation (hpi) by laser scanning confocal microscopy following $30 \mathrm{~min}$ of MDC staining. Scale bars are $10 \mu \mathrm{m}$. C, Top panel, localization of GFP:Atg8 in appressoria on hydrophobic surfaces at 24 hpi. Bottom panel, localization of GFP:Atg8 in germinating spores at 24 hpi on hydrophilic surfaces following the indicated spore treatments at 0 hpi. NT = no treatment; $\mathrm{AM}=400 \mathrm{nM}$ amiodarone chloride; Rap = $200 \mathrm{nM}$ rapamycin; cAMP = 10 mM monobutyryl cyclic AMP. Germ tube GFP:Atg8 puncta are indicated by arrows. Images are representatives of 50 germinating spores repeated in triplicate. Scale bars are $10 \mu \mathrm{m}$. D, Quantification of GFP:Atg8 puncta in germ tubes on hydrophilic surfaces at 24 hpi following the indicated spore treatments at 0 hpi. Bars are the mean number of GFP:Atg8 puncta observed in germinating spores. Error bars indicate standard deviation $(\mathrm{SD})$. Different letters indicate least significant difference $(\mathrm{LSD}$, $\alpha=0.05)$. E, Activation of autophagy by AM treatment at 0 hpi induced nuclear degeneration in germinating WT H1:RFP spores on hydrophilic surfaces. Images are representatives of 100 germinating spores repeated in triplicate. Scale bars are $10 \mu \mathrm{m}$. F, Bars are the mean numbers of nuclei observed in germinating spores at 24 hpi with (AM) or without (NT) amiodarone chloride treatment at $0 \mathrm{hpi}$ on hydrophilic surfaces. Error bars indicate standard deviation (SD). Different letters indicate least significant difference (LSD, $\alpha=0.05)$. G, SYTO green-stained nuclei in germinating spores of the $\Delta a t g 8$ mutant strain on the indicated surfaces at 24 hpi. Scale bars are $10 \mu \mathrm{m}$. 
spores on hydrophilic surfaces, by $24 \mathrm{hpi}$, resembled germinating $\Delta a b l 1$ spores on hydrophobic surfaces (which themselves resembled glucose-treated WT spores on hydrophobic surfaces).

Germ tube morphotype plasticity requires cPKA.

We next determined that morphotype plasticity on hydrophobic surfaces (either in WT spores following glucose treatment or in $\Delta a b l l$ spores without treatment), and its restriction on hydrophilic surfaces, was dependent on cPKA. We hypothesized that mechanisms governing morphotype plasticity under $\mathrm{TOR}_{\mathrm{on}}$ conditions (i.e., in the absence of $A B L 1$ or in WT in the presence of exogenous glucose) must act upstream of TOR. Our reasoning was that TOR acts downstream of cPKA and promotes appressorium formation (type IV morphotype) when TOR signaling is inactive and cAMP/PKA signaling is active $\left(\mathrm{TOR}_{\text {off }}\right.$ and $\mathrm{cAMP} / \mathrm{PKA}_{\text {on }}$ ) (Marroquin-Guzman and Wilson 2015; Marroquin-Guzman et al. 2017b). Conversely,
A

Wydrophobic surface QI 8 II QIII DIV

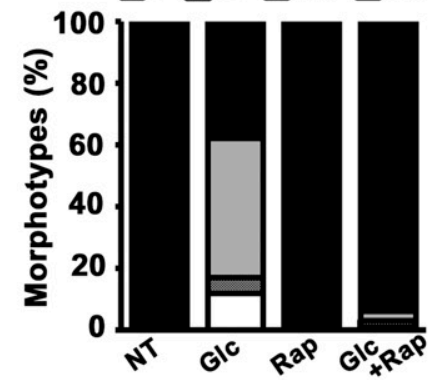

B

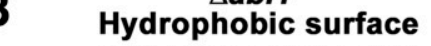
III DIII DIV

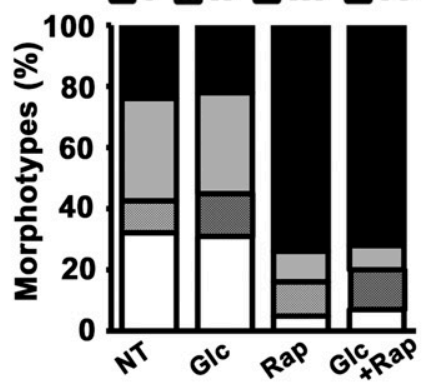

WT

Hydrophilic surface I III QIII DIV

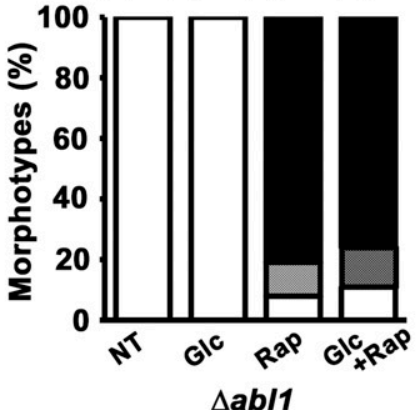

Hydrophilic surface I III םIII

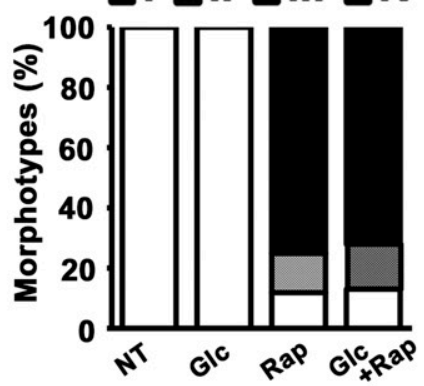

C

$\triangle a b / 1$ H1:RFP
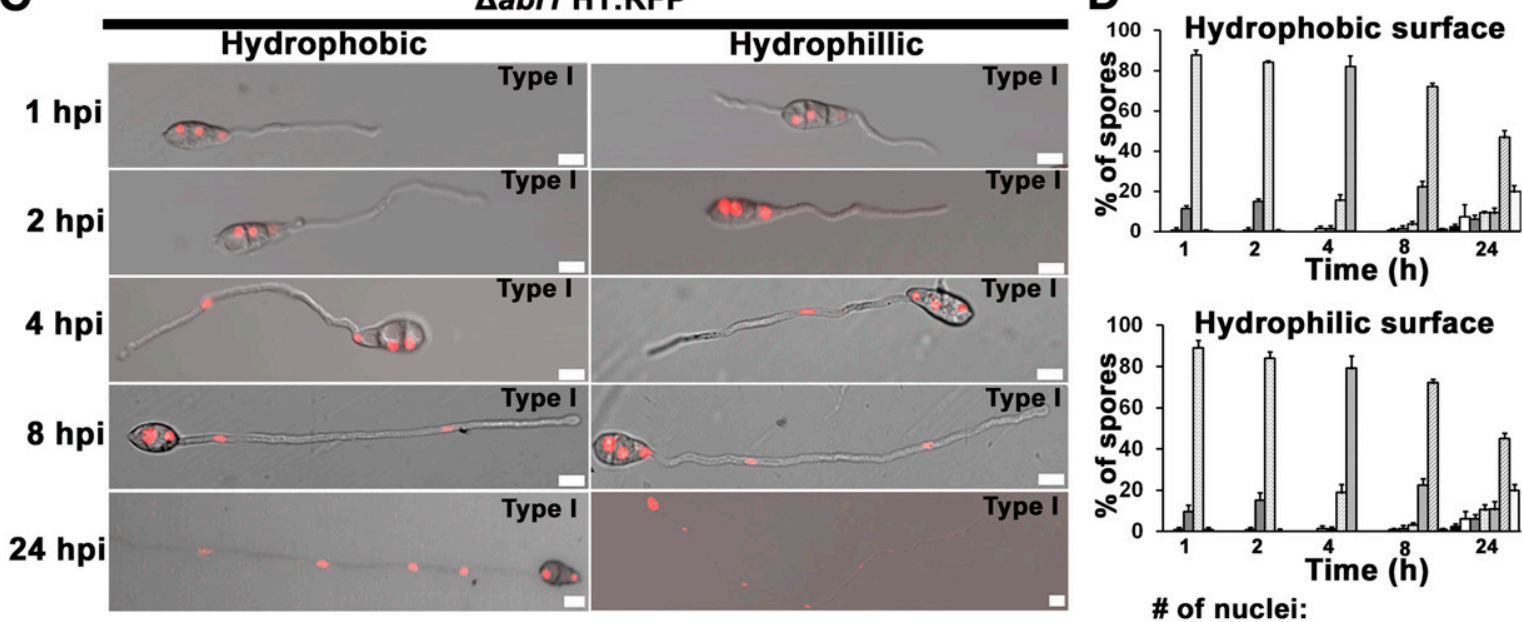

E Hydrophobic surface

Hydrophilic surface
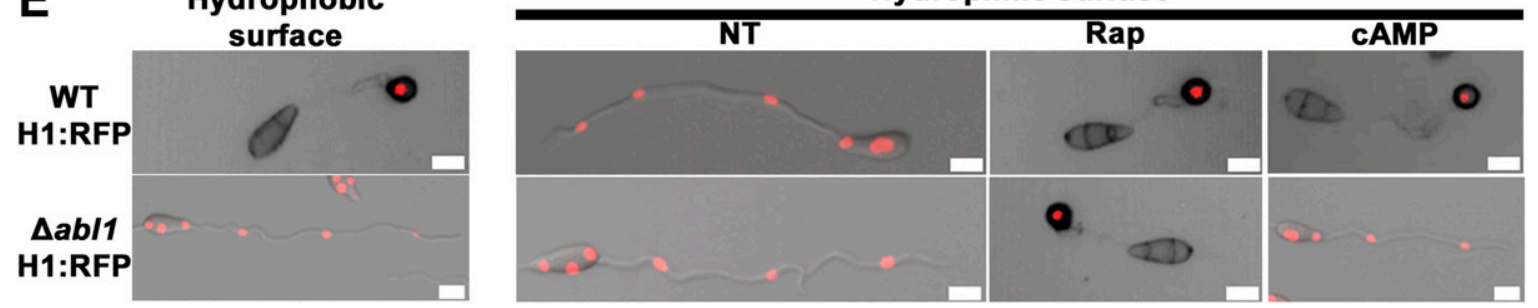
type I to IV morphotype plasticity occurs when TOR signaling and cAMP/PKA signaling pathways are both active TOR $_{\text {on }}$ and $\mathrm{cAMP} / \mathrm{PKA}_{\mathrm{on}}$ ) following the germination of $\triangle a b l l$ spores, or glucose-treated WT spores, on hydrophobic surfaces (Marroquin-Guzman et al. 2017b). Neither appressorium formation nor morphotype plasticity is supported under conditions where TOR signaling is active and cAMP/PKA signaling is inactive $\left(\mathrm{TOR}_{\text {on }}\right.$ and $\mathrm{cAMP} / \mathrm{PKA}_{\text {off }}$ ), i.e., during $\mathrm{WT}$ and $\triangle a b l 1$ spore germination on hydrophilic surfaces (Fig. 3A and B). Thus, morphotype plasticity depends on active cAMP/PKA signaling in the context of active TOR signaling. To confirm that cAMP/PKA signaling contributes to morphotype plasticity when TOR signaling is active, we created a $\Delta a b l 1 \Delta c p k a$ double mutant strain. As shown in Figure 4A and B, morphotype diversity was abolished in the $\Delta a b l 1 \Delta c p k a$ double mutant compared with $\Delta a b l l$ and glucose-treated WT spores on hydrophobic surfaces. Germinating $\Delta a b l 1 \Delta c p k a$ spores produced only type I morphotypes regardless of exogenous glucose treatment, but appressorium morphogenesis was mostly remediated by Rap treatment (Fig. 4A and B).

As previously reported (Xu et al. 1997), the $\Delta c p k a$ single mutant control strain produced nonfunctional appressoria on nutrient-free hydrophobic surfaces that were delayed in formation compared with WT (Supplementary Fig. S2). Nonetheless, as shown in Figure 4A loss of CPKA alone was sufficient to almost entirely abolish morphotype diversity on glucose-rich hydrophobic surfaces. When considered together, we interpret these results to indicate that early steps in the cAMP/PKA signaling pathway-from cPKA until the downstream point at which the cAMP/PKA pathway is inhibited by $\mathrm{TOR}_{\mathrm{on}}$ - contribute to morphotype plasticity under $\mathrm{TOR}_{\text {on }}$ and cAMP/PKA ${ }_{\text {on }}$ conditions (Fig. 4C).

\section{Appressorial morphogenesis and conidial nuclear degradation on hydrophilic surfaces requires $\mathrm{G} 2$ arrest and intact CAMP/PKA signaling.}

To gain more insight into the relationships between TOR, cAMP/PKA, autophagy, and mitosis during spore germination under noninductive conditions, we next asked whether, as on hydrophobic surfaces, G2 arrest was sufficient to induce appressorial morphogenesis on hydrophilic surfaces. We subsequently discovered that it was not.

On hydrophobic surfaces, $\mathrm{TOR}_{\text {off }}$ is required to arrest mitosis in $\mathrm{G} 2$ in order to initiate appressorium morphogenesis, because treating $\triangle a b l l$ spores (which are constitutive for $\mathrm{TOR}_{\mathrm{on}}$ ) with the G2 inhibitor benomyl (Ben) resulted in the concomitant induction of appressoria and autophagy on hydrophobic surfaces (Marroquin-Guzman et al. 2017b). To understand whether Ben treatment affected appressorial morphogenesis on noninductive hydrophilic surfaces, we treated spores of WT H1: RFP and $\Delta a b l 1 \mathrm{H} 1:$ RFP strains with $30 \mu \mathrm{M}$ Ben at 0 hpi and applied them to nutrient-free hydrophobic and hydrophilic surfaces. When applied to hydrophobic surfaces, by $24 \mathrm{hpi}$, as previously reported (Marroquin-Guzman et al. 2017b), Ben treatment promoted appressorium formation by germinating $\triangle a b l 1 \mathrm{H1}:$ RFP spores, did not inhibit appressorium formation by WT H1:RFP spores, and strongly or constitutively induced autophagy in both $\triangle a b l 1 \mathrm{H} 1$ :RFP and WT H1:RFP (Fig. 5A). This latter effect, itself resulting from an inability to progress through $\mathrm{G} 2$, results in complete nuclear degradation, as previously described (Marroquin-Guzman et al. 2017b). In contrast, on hydrophilic surfaces Ben treatment at 0 hpi did not induce appressoria formation or autophagy by germinating WT H1:RFP or $\triangle a b l 1 \mathrm{H} 1$ :RFP spores, although mitosis was arrested compared with the untreated controls (Fig. 5A). To account for the loss of appressorium formation following $\mathrm{G} 2$ arrest by Ben on hydrophilic surfaces, we considered that, whereas on hydrophobic surfaces cAMP/PKA signaling was active, on hydrophilic surfaces cAMP/PKA signaling was inactive. To determine whether cAMP/PKA signaling was required for appressorium formation in addition to G2 arrest, we treated WT $\mathrm{H} 1: \mathrm{RFP}$ and $\triangle a b l 1 \mathrm{H} 1: \mathrm{RFP}$ spores with both $30 \mu \mathrm{M}$ Ben and $10 \mathrm{mM}$ cAMP. For germinating $\triangle a b l 1 \mathrm{H} 1$ :RFP spores we determined that, whereas Ben treatment alone (Fig. 5A) or cAMP treatment alone (Fig. 3E) could not induce appressoria in germinating $\triangle a b l 1 \mathrm{H} 1: \mathrm{RFP}$ spores by $24 \mathrm{hpi}$, both treatments added to spores together at 0 hpi induced appressoria formation and strong autophagy (resulting in complete nuclear degradation) in this mutant strain (Fig. 5A). Thus, this is the first condition where cAMP was shown to affect appressoria formation by $\Delta a b l 1$ strains. Dual Ben + cAMP treatments also induced appressoria formation in germinating WT H1:RFP spores on hydrophilic surfaces. Furthermore, we determined G2 arrest and cAMP/PKA signaling were not order-dependent, because staggered Ben and cAMP treatments stimulated appressorial morphogenesis in both WT H1:RFP and $\triangle a b l 1 \mathrm{H} 1$ : RFP strains on hydrophilic surfaces regardless of whether Ben or cAMP was added first (Fig. 5B, bottom panel). Our interpretation of these results is that both G2 arrest and cAMP/PKA are required, but neither alone is sufficient, for appressorium morphogenesis (Fig. 5C). Consequently, neither active cAMP/PKA signaling in the absence of $\mathrm{G} 2$ arrest (i.e., $\triangle a b l l$ on hydrophobic surfaces or $\Delta a b l l$ on hydrophilic surfaces following cAMP treatment) nor G2 arrest in the absence of cAMP/PKA signaling (i.e., Ben treatment of germinating spores on hydrophilic surfaces) can induce appressorium formation.

The results presented here suggest that although G2 inhibits downstream cAMP/PKA signaling, the absence of this inhibition following G2 arrest cannot by itself activate downstream cAMP/PKA signaling if this pathway is inactive. Further, these results provide detail on how $\mathrm{TOR}_{\mathrm{on}}$ inhibits cAMP/PKA signaling by indicating it likely occurs via the TOR-dependent metabolic checkpoint at G2, rather than through direct interactions of TOR with downstream components of the cAMP/PKA pathway (Fig. 5C). Finally, we note

Fig. 3. Hydrophilic surfaces restrict wild-type (WT) and $\Delta$ abl1 germ tube morphotype plasticity. A, Proportion of morphotypes formed at 24 h postinoculation (hpi) by germinating WT spores under the indicated conditions. Type I morphotype is a long germ tube with no appressorium at the tip; type II is a long germ tube with an appressorium; type III is a short germ tube with a tip swelling and no appressoria; type IV is a short germ tube with an appressorium at the tip. Bars are the average of each morphotype, at $24 \mathrm{hpi}$, formed by 50 germinating spores repeated in triplicate, with six technical replicates per biological replicate. Treatments were added at 0 hpi. B, Germinating $\Delta a b l 1$ spores, which are constitutively activated for TOR, were restricted to forming the type I morphotype on noninducible hydrophilic surfaces (with or without glucose treatment) compared with germinating $\Delta a b l 1$ spores on hydrophobic surfaces. Bars are the average of each morphotype, at $24 \mathrm{hpi}$, formed by 50 germinating spores repeated in triplicate, with six technical replicates per biological replicate. Treatments were added at 0 hpi. C, type I morphotype formed by $100 \%$ of $\Delta a b l 1$ histone H1 fused to red fluorescent protein (H1:RFP) spores germinating on hydrophilic surfaces resembled the type I morphotype formed by $32 \%$ of germinating $\triangle a b l 1 \mathrm{H} 1: \mathrm{RFP}$ spores on hydrophobic surfaces. This included multiple rounds of mitosis in germ tubes and abolishment of conidial nuclear degradation. D, Average number of nuclei carried by germinating $\Delta a b l 1$ H1:RFP spores on hydrophobic and hydrophilic surfaces at indicated time points. Bars are the average number of nuclei carried by 100 germinating spores repeated in triplicate. Error bars are SD. E, Treatment with 100 to $200 \mathrm{nM}$ rapamycin (Rap) but not $10 \mathrm{mM}$ monobutyryl cyclic AMP (cAMP) restored appressorium formation by $\triangle a b l 1 \mathrm{H} 1:$ RFP strains on hydrophilic surfaces. NT = no treatment; Glc $=1 \%$ (wt/vol) glucose; Rap $=100$ to $200 \mathrm{nM}$ rapamycin. C and E, Scale bars are $10 \mu \mathrm{m}$. 
that in WT on hydrophilic surfaces, although Ben treatment alone is not sufficient to induce appressorium formation, cAMP treatment alone is sufficient. This hints at a connection between cAMP and G2 arrest that is not present in $\Delta a b l 1$ germinating spores and, thus, might involve Abll and TOR. This connection is elaborated in more detail below.

Taken together, we conclude that TOR-dependent G2 arrest permits cAMP/PKA-signaling to induce autophagy and appressorium formation and, thus, progress the cell cycle through mitosis to G1/G0.

Loss of cAMP/PKA signaling results in multiple rounds of germ tube mitosis under inductive, hydrophobic conditions.

Our investigations of TOR and cAMP/PKA activity led us to conclude that $\mathrm{TOR}_{\text {off }}$-dependent $\mathrm{G} 2$ arrest and cAMP/PKA signaling are both necessary but that neither alone is sufficient to permit appressorial and autophagy induction on hydrophilic surfaces. To elaborate further on the connections between TOR and cAMP/PKA signaling, we next studied cell cycle progression and autophagy during spore germination in $\Delta m a c l$ strains that are abolished for cAMP/PKA signaling. We subsequently discovered that $\mathrm{TOR}_{\mathrm{off}}$-dependent $\mathrm{G} 2$ arrest does not occur, or occurs only transiently, if cAMP/PKA signaling is inactive during spore germination on otherwise inductive hydrophobic surfaces, thus indicating a more extensive degree of crosstalk between the TOR and cAMP/PKA pathways than hitherto was appreciated.

We generated a new $\triangle m a c 1$ mutant strain lacking the $M A C 1$ gene encoding adenylate cyclase and assessed germinating $\Delta$ macl spores on hydrophobic surfaces for morphotype plasticity, cell cycle regulation, and response to Rap and cAMP. Unlike $\Delta c p k a, \Delta m a c l$ mutant strains do not form any appressorial structures (Choi and Dean 1997). The loss of MAC1 restricted morphotype diversity, with germinating $\Delta m a c 1$ spores forming either long germ tubes without appressoria (type I) or short germ tubes without appressoria (type III) on all surfaces tested, including in the presence of glucose (Fig. 6A). This was consistent with our earlier observations that cAMP/PKA signaling through cPKA until its inhibition by $\mathrm{TOR}_{\mathrm{on}}$ is required for morphotype plasticity. As expected based on previous work, cAMP treatment resolved appressorium formation, but Rap treatment, for the first time, was shown to not remediate appressorium morphogenesis in this mutant strain (Fig. 6B).

Using SYTO green fluorescent nucleic acid staining, we were surprised to find that untreated germinating $\Delta$ macl spores on hydrophobic surfaces were abolished for autophagic cell death of conidial nuclei and carried multiple nuclei in germ tubes by 24 hpi (Fig. 6C). These results indicate that G2 arrest is abrogated in germinating $\triangle m a c l$ spores on hydrophobic surfaces, suggesting that $\mathrm{TOR}_{\text {off }}$-dependent $\mathrm{G} 2$ arrest (or the
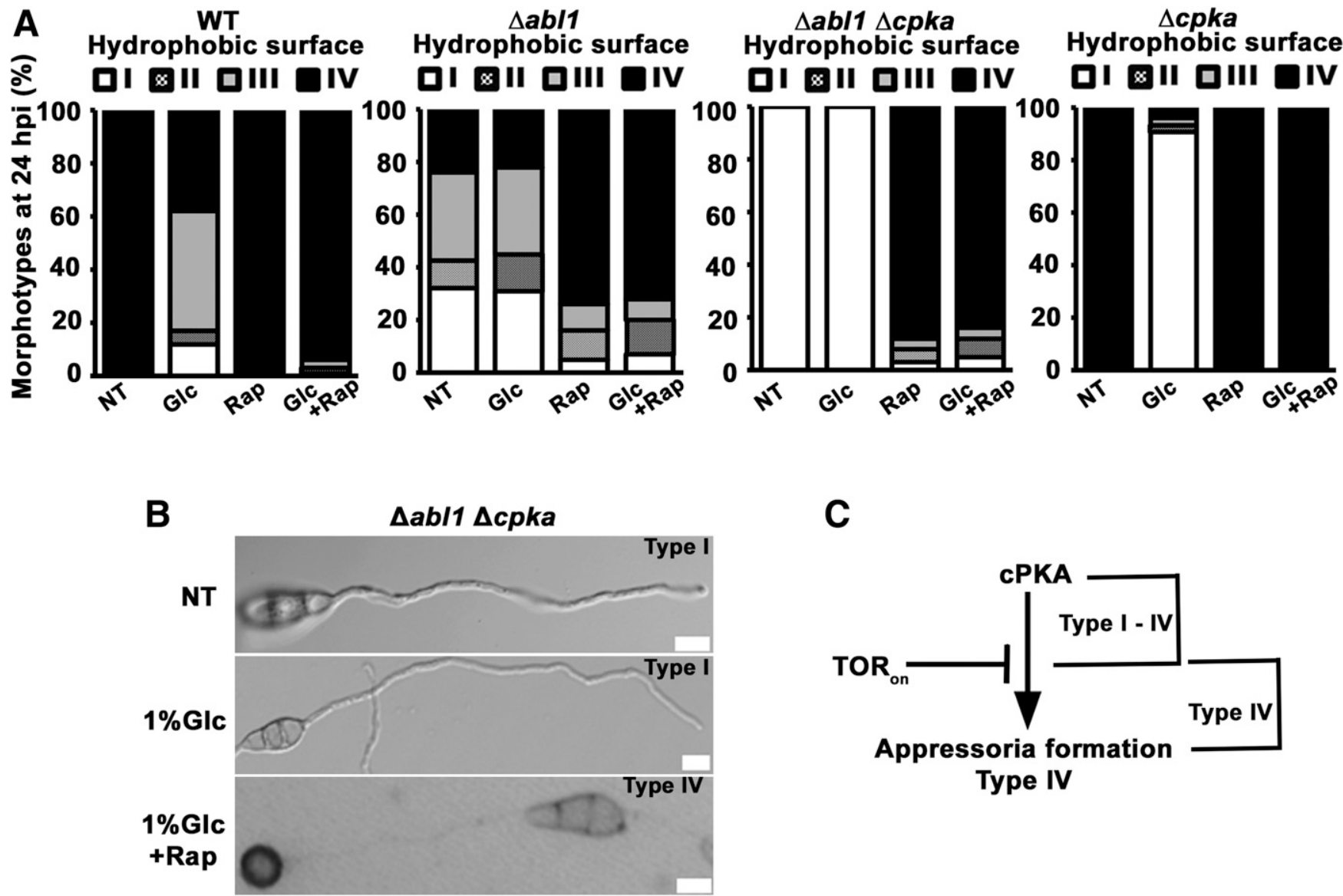

Fig. 4. cPKA is required for morphotype plasticity. A, Proportion of morphotypes formed at 24 h postinoculation (hpi) on hydrophobic surfaces by germinating spores of wild type (WT), $\Delta a b l 1, \Delta a b l 1 \Delta c p k a$, and $\Delta c p k a$ strains following the indicated treatments. Bars are the average of each morphotype, at 24 hpi, formed by 50 germinating spores repeated in triplicate, with six technical replicates per biological replicate. Type I morphotype is a long germ tube with no appressorium at the tip; type II is a long germ tube with an appressorium; type III is a short germ tube with a tip swelling and no appressoria; type IV is a short germ tube with an appressorium at the tip. B, Examples of morphotypes displayed at 24 hpi by the $\Delta a b l 1 \Delta c p k a$ double mutant following treatments on hydrophobic surfaces. Scale bars are $10 \mu \mathrm{m}$. C, Model showing relationship between cPKA, TOR signaling, morphotype plasticity, and appressorium formation. $\mathrm{NT}=$ no treatment; Glc $=1 \%(\mathrm{wt} / \mathrm{vol})$ glucose; Rap $=100$ to $200 \mathrm{nM}$ rapamycin. Treatments were added at 0 hpi. 
prevention of transient arrest) on hydrophobic surfaces requires either cAMP/PKA signaling or cAMP itself and that such a cAMP-dependent signal must act via Abl1, because $\Delta a b l 1$ mutants do not respond to cAMP (Fig. 3E). Similar cell cycle outcomes were observed for untreated and Rap- or
cAMP-treated germinating $\Delta m a c 1$ spores on hydrophilic surfaces (Fig. 6C). SYTO green fluorescent nucleic acid staining showed that cAMP treatment at $0 \mathrm{hpi}$ resulted in a single appressorial nucleus by $24 \mathrm{hpi}$, whereas $\mathrm{TOR}_{\text {off }}$ following Rap treatment arrested mitosis, likely in G2, but did
A

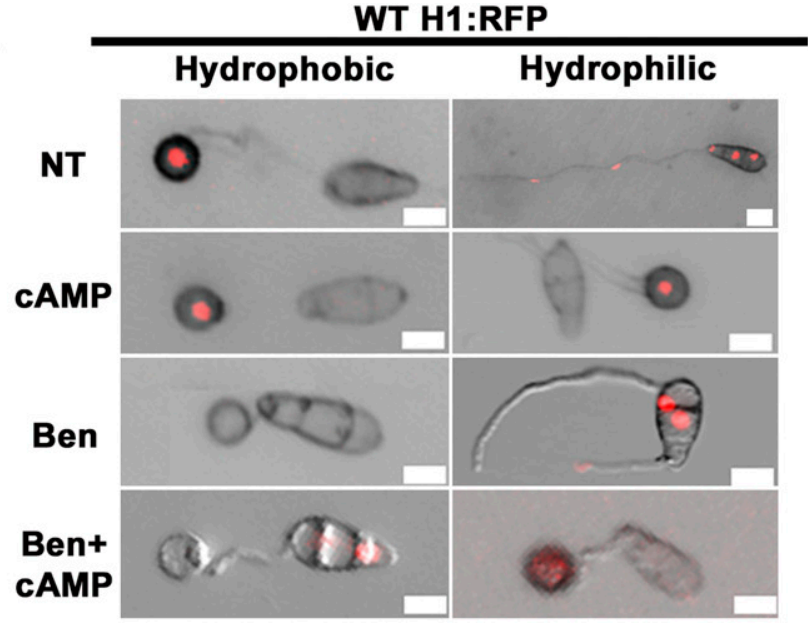

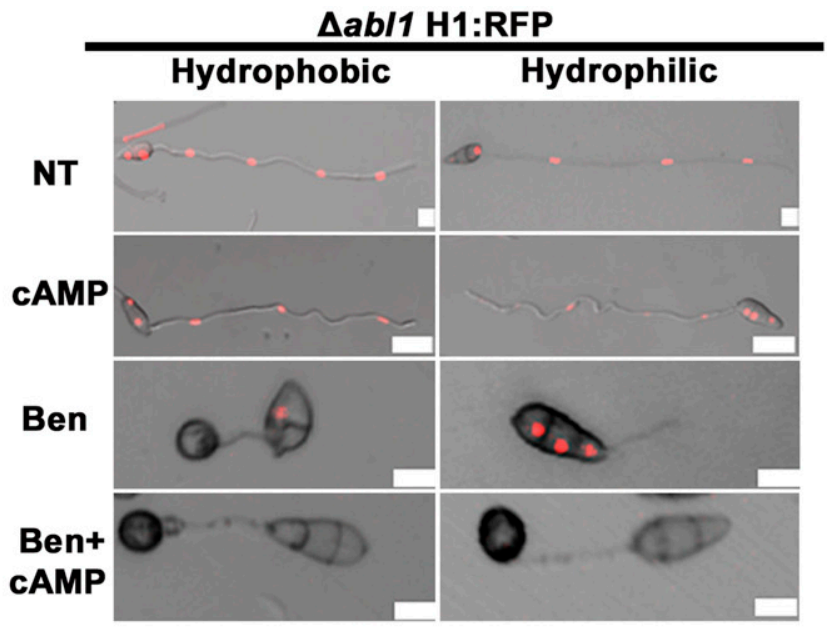

B

Hydrophilic surface
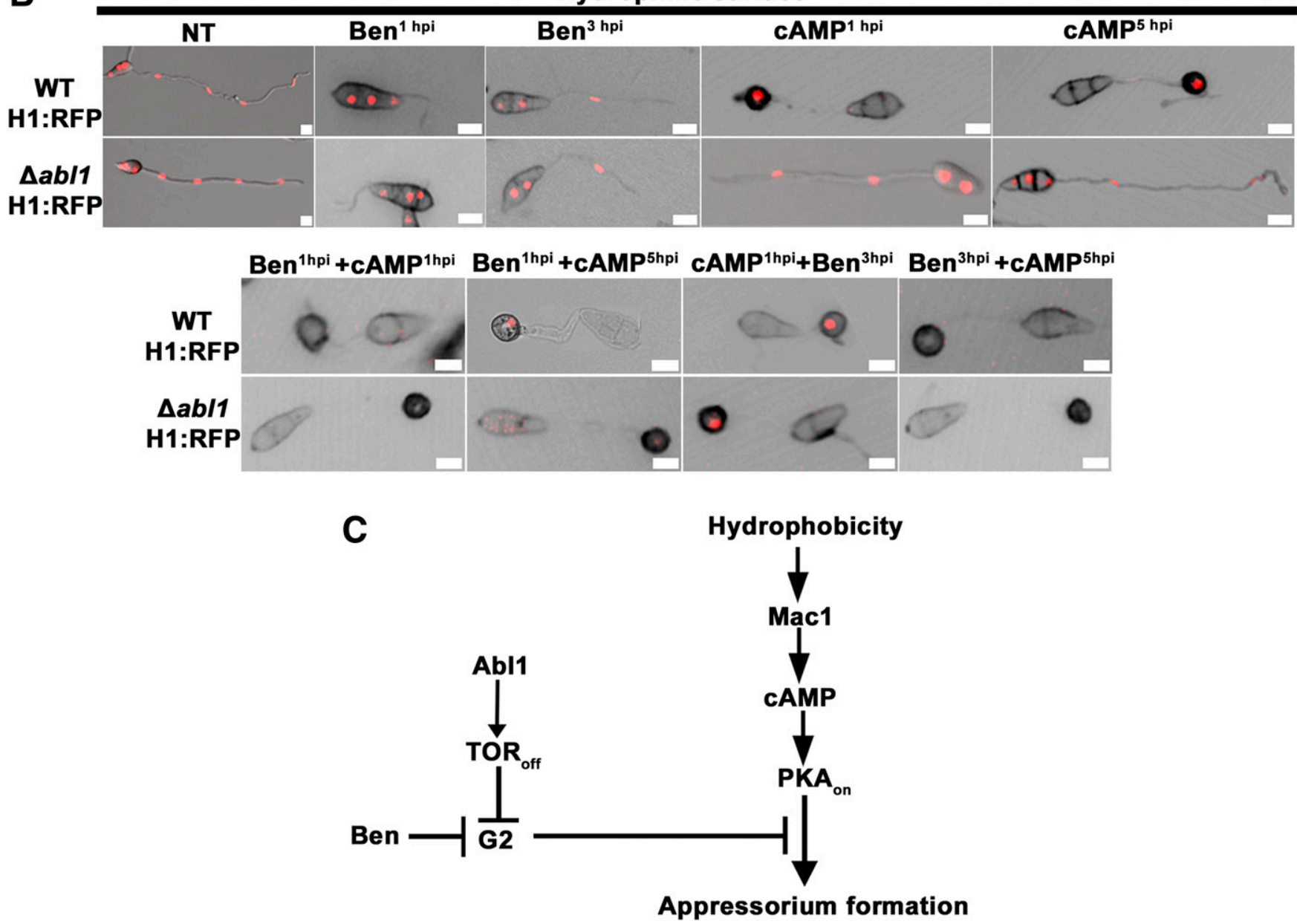

Fig. 5. G2 arrest and active cAMP/PKA signaling induce appressorium and autophagy. A, G2 arrest and cAMP/PKA signaling are both required for appressoria formation and autophagy. Treatments were added at $0 \mathrm{~h}$ postinoculation (hpi); images were taken at 24 hpi. B, Appressorium formation was order-independent with regard to G2 arrest and cAMP/PKA signaling. If active cAMP/PKA signaling is blocked by G2, G2 arrest by Ben will result in appressoria formation. If Ben arrests G2, but cAMP/PKA signaling is inactive, appressoria will not form until cAMP is added. Superscripts indicate treatment time; images were viewed at 24 hpi. C, Model depicting relationships between $\mathrm{G} 2$ arrest, $\mathrm{cAMP} / \mathrm{PKA}$ signaling, and appressorium formation. $\mathrm{A}$ and $\mathrm{B}, \mathrm{NT}=$ no treatment; Ben $=30 \mu \mathrm{M}$ benomyl; cAMP $=10 \mathrm{mM}$ monobutyryl cyclic AMP. Images represent major phenotypes $(>80 \%)$ of 50 germinating spores by 24 hpi. Experiments were performed in triplicate. Scale bars are $10 \mu \mathrm{m}$. 
A

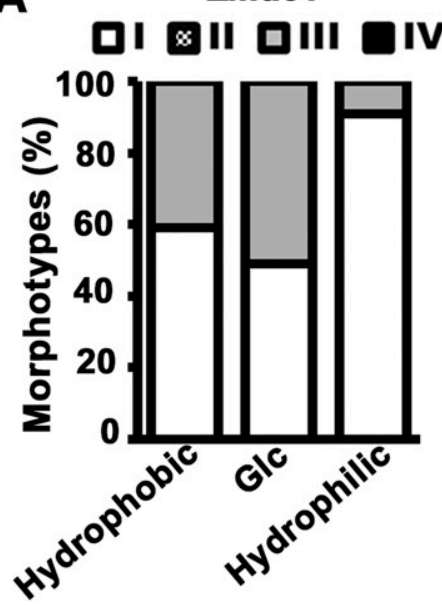

E

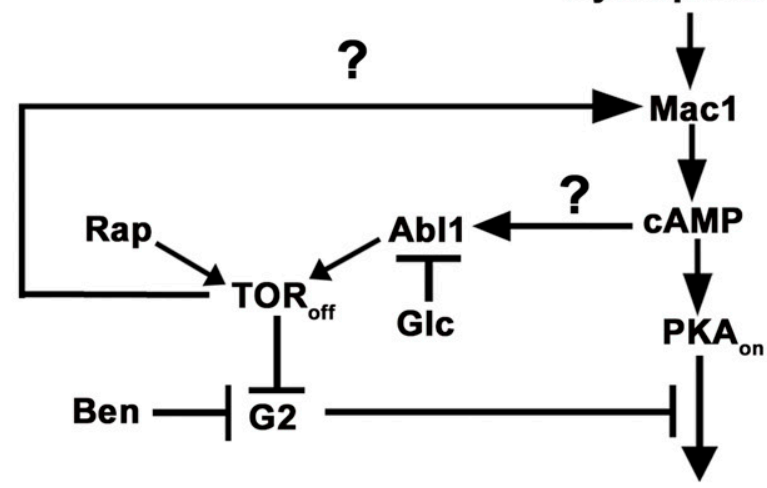

Appressoria formation

B

Hydrophobic surface at $24 \mathrm{hpi}$

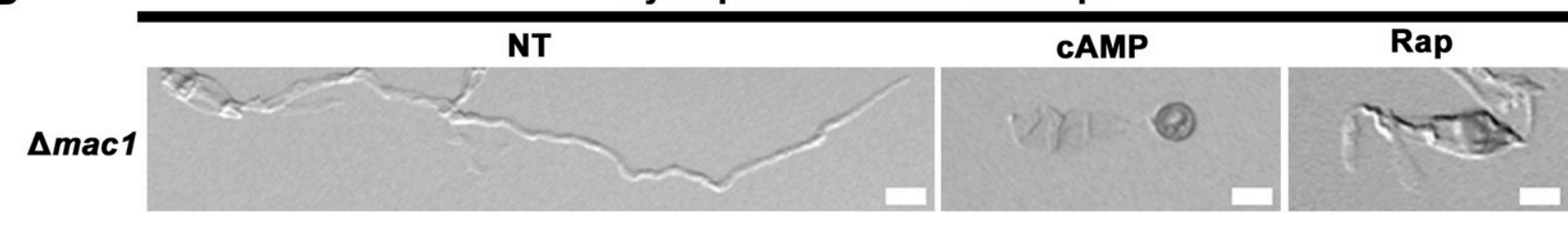

C

Hydrophobic surface at $24 \mathrm{hpi}$

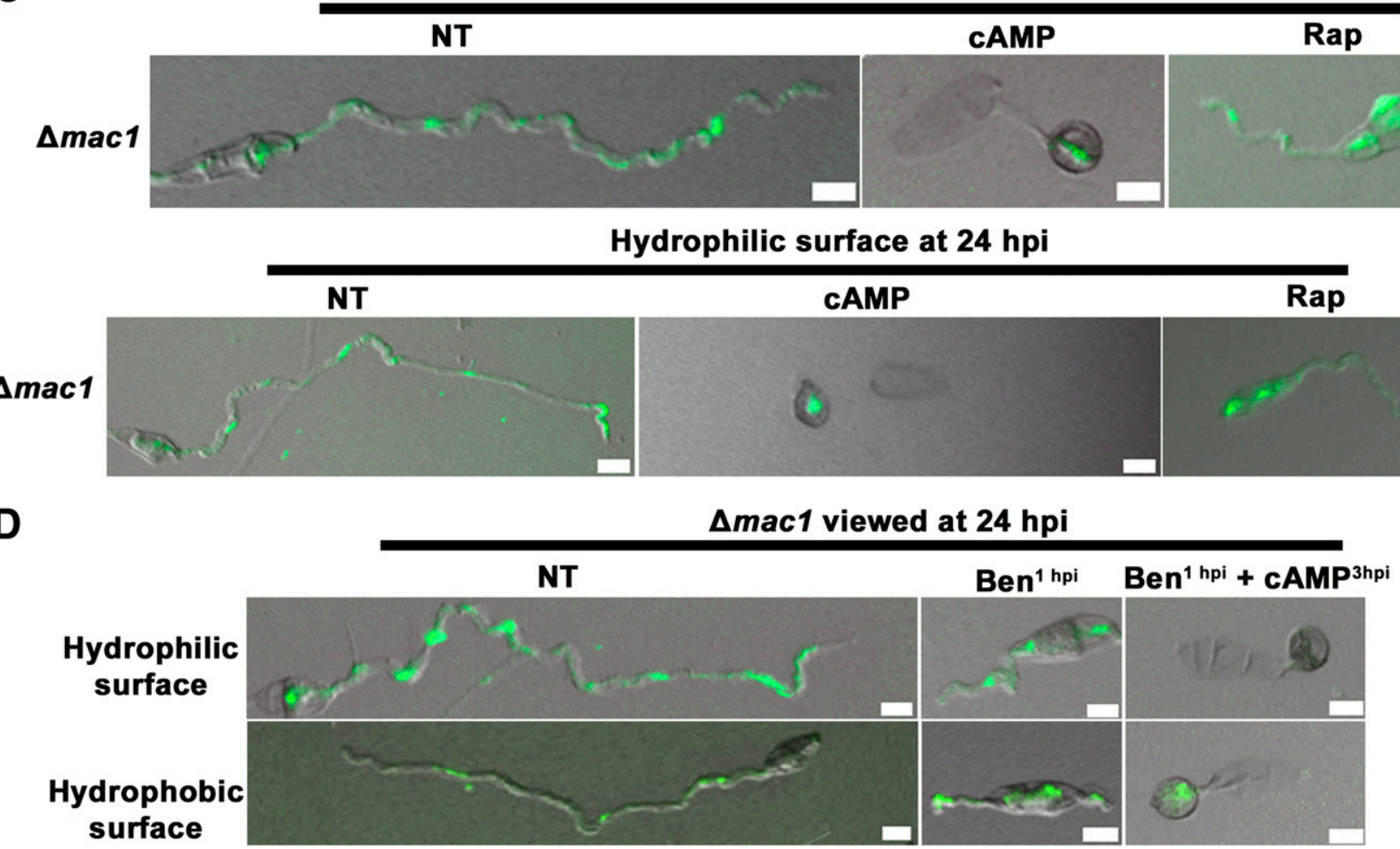

Fig. 6. Loss of cAMP/PKA signaling abrogates G2 arrest and prevents autophagy in $\Delta m a c 1$ germinating spores. A, Morphotype plasticity was reduced in germinating $\Delta$ mac1 spores, which by $24 \mathrm{~h}$ postinoculation (hpi) formed only type I (a long germ tube with no appressorium at the tip) and type III (a short germ tube with a tip swelling and no appressoria) morphotypes regardless of surface hydrophobicity or glucose treatment. Bars are the average number of each morphotype, at $24 \mathrm{hpi}$, formed by 50 germinating spores repeated in triplicate, with six technical replicates per biological replicate. Glc $=1 \%$ (wt/vol) glucose added at 0 hpi. B, cAMP, but not rapamycin (Rap), treatment at 0 hpi could restore appressorium formation by germinating $\Delta m a c 1$ spores. $\mathbf{C}$, Germinating spores of $\Delta$ macl underwent multiple rounds of germ tube mitosis and were abolished for autophagy and appressorium induction regardless of surface hydrophobicity. cAMP, but not Rap, treatment at 0 hpi could restore appressorium formation and autophagy by 24 hpi. D, G2 arrest by benomyl (Ben) treatment was not sufficient to induce appressoria formation by germinating $\Delta$ macl spores unless also accompanied by active cAMP/PKA signaling following cAMP treatment. Superscripts indicate treatment times. E, Model depicting relationships between the TOR- and cAMP/PKA-signaling pathways, G2 arrest, and appressorium formation. Question marks indicate inferred direct or indirect interactions between pathway components. B to D, NT = no treatment. Scale bars are $10 \mu \mathrm{m}$ 
not induce appressorium formation or autophagy (Fig. 6C). This indicates that in the absence of active cAMP/PKA signaling, $\mathrm{TOR}_{\mathrm{off}}$ alone is not sufficient to induce appressoria, which is consistent with the results shown in Figure 5.

Further support for the notion that in order to form appressoria $\mathrm{TOR}_{\text {off }}$-dependent $\mathrm{G} 2$ arrest requires cAMP/PKA signaling to progress to autophagy and appressorial morphogenesis is shown in Figure 6D, whereby Ben treatment of germinating $\Delta$ macl spores, on either hydrophilic or hydrophobic surfaces, arrested G2 and prevented multiple rounds of mitosis, but was not sufficient to induce autophagy and appressorium formation unless cAMP was also added. This indicates that cAMP/PKA signaling, when active, is required for autophagy and appressoria induction.

We conclude that the loss of MACl mimics hydrophilic conditions on hydrophobic surfaces with regard to the loss of autophagy control, loss of G2 arrest, and loss of morphotype plasticity during spore germination. We propose that our data support the model shown in Figure $6 \mathrm{E}$ and provide the first evidence for bidirectional crosstalk between TOR and cAMP/PKA signaling. In this model, cAMP/PKA signaling activates autophagy and appressorium morphogenesis, but only following $\mathrm{TOR}_{\text {off }}$-dependent $\mathrm{G} 2$ arrest. $\mathrm{TOR}_{\text {off }}$ requires active Abl1, and Abl1 is inhibited by glucose (Marroquin-Guzman et al. 2017b). However, because the loss of MAC1 results in multiple rounds of mitosis on hydrophobic surfaces, like $\triangle a b l 1$ (but without the morphotypes), this implies cAMP (directly or indirectly) activates Abl1 to inhibit TOR signaling. Furthermore, because Rap treatment arrested mitosis in $\Delta m a c 1$ germinating spores on hydrophilic surfaces but did not induce appressorium formation, this indicates $\mathrm{TOR}_{\text {off }}$ (directly or indirectly) activates Mac1. These results describe a novel feedforward subnetwork driving appressorium formation under $\mathrm{TOR}_{\text {off }}$ and cAMP/PKA ${ }_{\text {on }}$ inductive conditions.

\section{Later rounds of germ tube mitosis are competent to form appressoria.}

We next asked if there was biological significance to the observation that germinating WT spores elicited the same responses regardless of unfavorable conditions (exogenous glucose or hydrophilic surfaces), namely the abrogation of G2 arrest, loss of autophagy, and multiple rounds of mitosis in germ tubes. We hypothesized that later rounds of mitosis might be competent to form appressoria. This was suggested by observations that $\Delta m a c l$ could be induced to form appressoria on hydrophobic surfaces following cAMP treatment for up to 12 hpi (Fig. 7A) despite the fact that treatments at later time points produced appressoria following substantial germ tube growth (Fig. 7B). This hypothesis was tested more precisely, and as shown in Figure 7C, G2 arrest in later rounds of mitosis can induce appressorial morphogenesis in WT if cAMP/PKA signaling is also active. Here, germ tube mitosis on hydrophilic surfaces was arrested at $10 \mathrm{hpi}$ in G1 (using cycloheximide $[\mathrm{CHX}]$ ), S-phase (using hydroxyurea [HU]), or G2 (using Ben). In all three cases, the cell cycle was arrested after three rounds of mitosis in the majority of germ tubes, but appressoria were not induced by these treatments alone. However, adding cAMP with Ben at 10 hpi (but not with $\mathrm{CHX}$ or HU) induced appressorium formation (Fig. 7C), indicating that the third round of mitosis was competent to form appressoria following G2 arrest by Ben and cAMP/PKA activation by exogenous cAMP. These results show dynamic changes in appressorial morphogenetic capacity are possible in response to changing environmental conditions.

To determine whether the relationship between Abl1, TOR, and appressoria formation was maintained during later rounds of mitosis, we added cAMP (Fig. 7D) or Rap (Fig. 7E) to germinating WT H1:RFP and $\Delta a b l 1 \mathrm{H} 1$ :RFP spores, on hydrophilic surfaces, at different time points and observed the effect on mitosis and appressorial morphogenesis by $24 \mathrm{hpi}$. $\triangle a b l 1 \mathrm{H} 1: \mathrm{RFP}$ spores did not produce appressoria in response to cAMP treatment at any time point, indicating $\mathrm{TOR}_{\mathrm{on}}$ resulting from $\triangle a b l 1$ inhibits $\mathrm{cAMP} / \mathrm{PKA}$ signaling downstream of cPKA during later rounds of mitosis. Germinating WT H1:RFP spores produced appressoria following cAMP treatments added up to at least $10 \mathrm{hpi}$ (Fig. 7D). For most germinating spores, treatment at 10 hpi ensured cAMP acted during or after the third round of mitosis following spore germination (Figs. 1B and 7C). These results provide more evidence that later cell cycle rounds are competent to form appressoria if conditions change and that appressorial morphogenesis in response to cAMP requires $A B L 1$ up to at least the third round of mitosis. However, it is notable that later cAMP treatments did not always induce autophagic cell death of the conidium, although germ tube nuclei were destroyed.

Rap treatment (Fig. 7E) induced appressorium formation by both WT H1:RFP and $\triangle a b l 1 \mathrm{H} 1$ :RFP germinating spores on hydrophilic surfaces, but only if added up to 4 hpi (i.e., before the second round of mitosis). The Rap treatment results are consistent with $A B L 1$-dependent $\mathrm{TOR}_{\text {off }}$, also resulting in alleviation of cAMP/PKA inhibition during later mitotic rounds. Differences in the temporal action of Rap and cAMP might indicate that cAMP/PKA- and TOR-signaling pathways become disengaged or are rewired at later time points, might indicate TOR becomes insensitive to Rap during later mitotic rounds, or might indicate Rap is not taken up by the cell at later time points.

When considered together, these results reveal the novel prospect that G2 arrest and cAMP/PKA signaling during any of at least the first three rounds of mitosis following spore germination are sufficient to induce appressoria morphogenesis and autophagy (as assessed by conidial nuclear degradation).

\section{Integrated network architecture.}

Our results described herein, when combined with our previous data, support the signaling architecture shown in Figure 8. This model shows how arresting in G2 induces autophagy concomitantly with appressorium formation, but only if cAMP/PKA signaling is active, resulting in $\mathrm{TOR}_{\mathrm{on}}$ and reprogression of the cell cycle through mitosis to $\mathrm{TOR}_{\mathrm{off}}$ and G1/G0 arrest of the appressorial nucleus (Marroquin-Guzman et al. 2017b). In the absence of G2 arrest, the cell cycle continues unabated, resulting in germ tube growth that may or may not be accompanied by morphotype plasticity depending on the activity of cPKA. TOR is likely intrinsically active (MarroquinGuzman et al. 2017b), and TOR inactivation is positively regulated by Abl1 or Rap. Glutamine (Gln) is a positive TOR activator and can override Abll but not Rap inhibition of TOR (Marroquin-Guzman and Wilson 2015). Abl1 inactivation is mediated by glucose or glucose-6-phosphate but not downstream metabolites (Marroquin-Guzman et al. 2017b). Abl1 activation (resulting in $\mathrm{TOR}_{\mathrm{off}}$ ) is suggested here, for the first time, to be mediated (directly or indirectly) by cAMP. cAMP control of Abl1 would account for why WT and $\Delta m a c 1$ but not $\Delta a b l 1$ germinating spores form appressoria on hydrophilic surfaces after cAMP treatment. cAMP control of Abl1 would also account for why TOR is active in WT on nutrient-free hydrophilic surfaces, suggesting that either the absence of cAMP or the presence of glucose/glucose-6-phosphate is sufficient to inactivate Abl1. Strikingly, because Rap treatment induced appressoria formation by germinating WT and $\Delta a b l 1$ spores on hydrophilic surfaces but only arrested $\Delta m a c l$ germ tube mitosis at G2 without promoting appressorium formation, our data also suggest the converse-that $\mathrm{TOR}_{\text {off }}$ mediates 
cAMP levels by (directly or indirectly) activating Mac1. G2 arrest by Ben similarly did not induce appressoria formation by germinating $\Delta m a c l$ spores, suggesting $\mathrm{TOR}_{\text {off }}$ activation of Mac1 is in addition to, and independent of, the role of TOR off $_{\text {in }}$ alleviating cAMP/PKA downstream inhibition via G2 arrest. Together, cAMP control of TOR via Abl1 and TOR control of cAMP via Mac1 suggests a feed-forward subnetwork emerging from the underlying signaling architecture. This subnetwork reinforces cell cycle tuning, autophagy activation, and appressorium formation when active under inductive conditions, but disrupts appressorium morphogenesis and promotes germ tube growth when inactivated by disparate noninductive conditions.

An alternative explanation that does not invoke cAMP control of Abl1 to account for the loss of G2 arrest in germinating

A

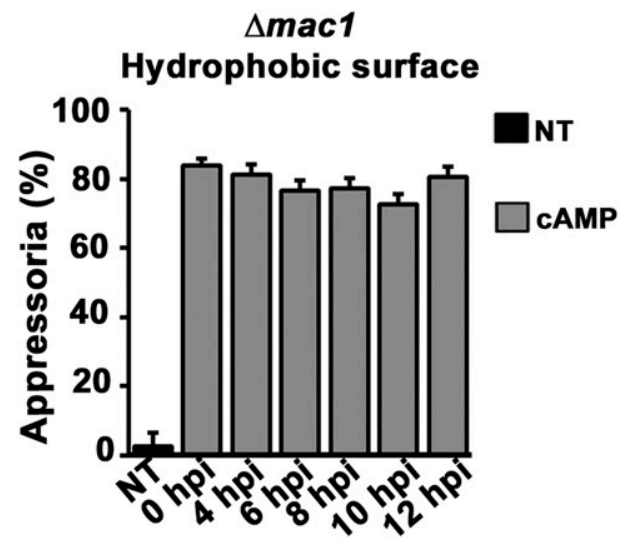

B $\Delta m a c 1$ on hydrophobic surfaces viewed at $24 \mathrm{hpi}$ after cAMP added at:

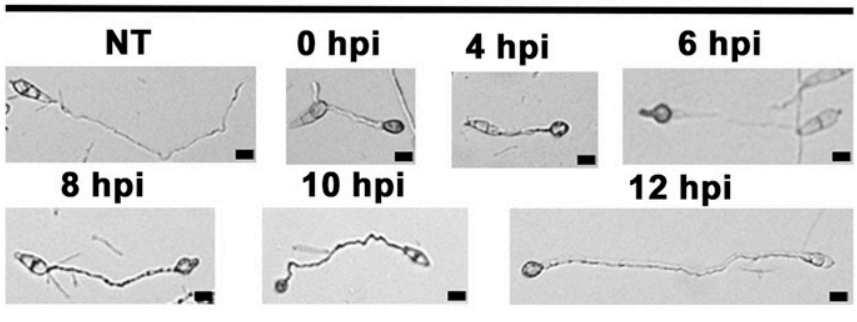

C

WT H1:RFP on hydrophilic surface viewed at $24 \mathrm{hpi}$

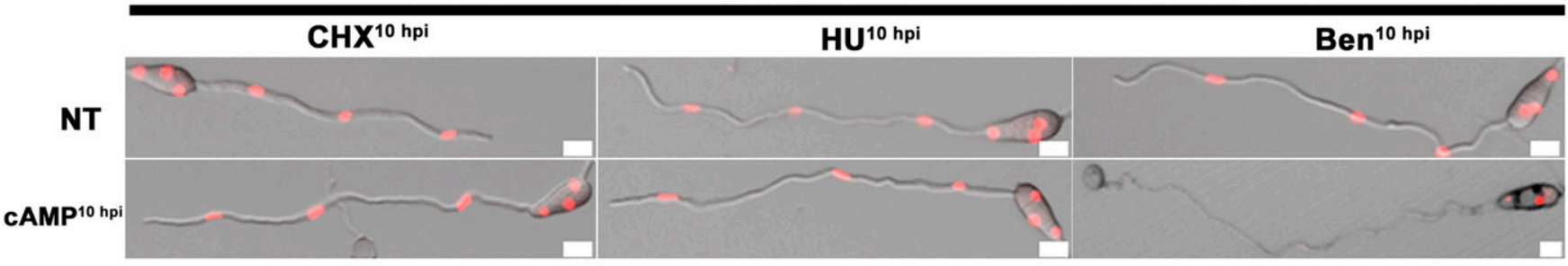

D

WT H1: RFP at $24 \mathrm{hpi}$

WT H1:RFP on hydrophilic surface at 24 hpi

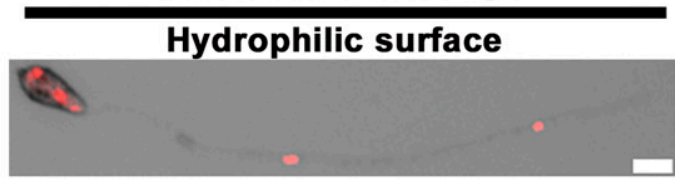

Hydrophobic surface
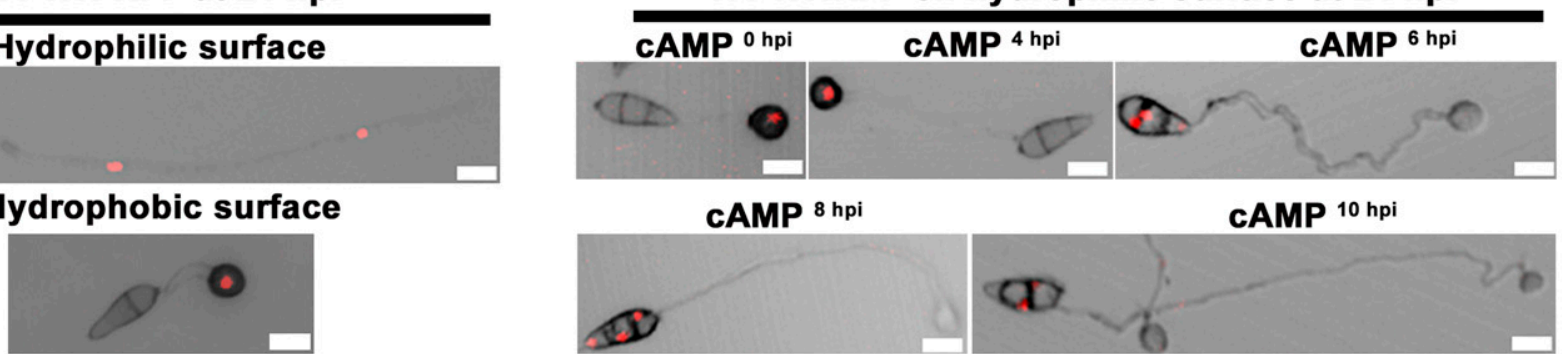

CAMP $8 \mathrm{hpi}$

CAMP $10 \mathrm{hpi}$

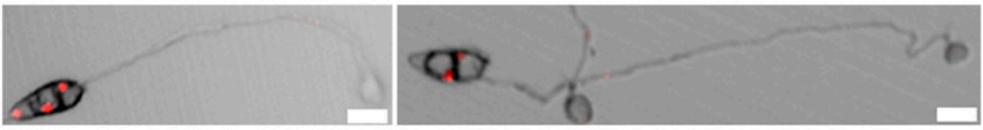

$\mathbf{E}$

Viewed at $\mathbf{2 4}$ hpi after Rap added at:

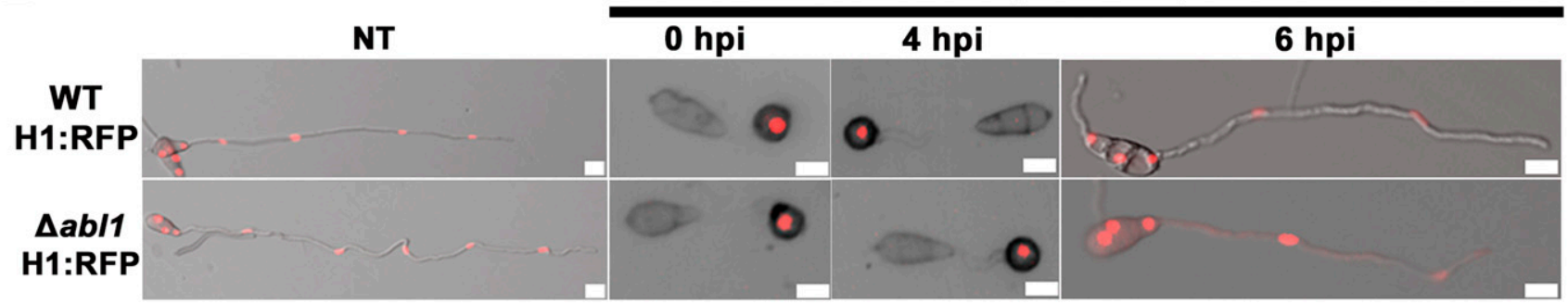

Fig. 7. Extended rounds of mitosis in germ tubes produce appressoria-competent nuclei. $\mathbf{A}$ and $\mathbf{B}$, Germinating $\Delta m a c l$ spores treated with cAMP at the indicated time points were restored for appressorium formation on hydrophobic surfaces. A, Bars are the average percentage of 50 germinating $\Delta m a c 1$ spores forming appressoria by $24 \mathrm{~h}$ postinoculation (hpi) on each of three coverslips. Error bars are standard deviation (SD). C, Active cAMP/PKA signaling and G2 arrest by benomyl (Ben), but not S-phase arrest by hydroxyurea (HU) or G1 arrest by cyclohexamide (CHX), induced appressoria formation and autophagy after the third round of mitosis in germinating wild-type (WT) spores on hydrophilic surfaces. Ben $=30 \mu \mathrm{M}$ benomyl; $\mathrm{HU}=50 \mathrm{mM}$ hydroxyurea; $\mathrm{CHX}=2 \mathrm{mM}$ cyclohexamide. Superscripts indicate treatment times. D, Exogenous cAMP treatments at indicated time points restored appressoria formation and autophagy in WT spores on hydrophilic surfaces when applied up to $10 \mathrm{hpi}$. E, Exogenous rapamycin (Rap) treatments restored appressorium formation and autophagy to germinating WT and $\Delta a b l l$ spores on hydrophilic surfaces when added up to 4 hpi. NT $=$ no treatment. B to D, Scale bars are $10 \mu \mathrm{m}$. 
$\Delta m a c 1$ spores is that in the absence of cAMP/PKA signaling, $\mathrm{TOR}_{\text {off }}$ might only transiently arrest in G2 before reactivating and rapidly progressing the cell cycle through mitosis to G1 and the next round of mitosis in germ tubes (rather than to G1/G0 in appressorial nuclei). Such reinforcement of $\mathrm{TOR}_{\text {off }}$ at $\mathrm{G} 2$ by cAMP/PKA signaling is a possible hypothesis that also explains the presented data. However, it is not, in our view, currently testable, and the most prudent and safe explanation at this time is that $\mathrm{TOR}_{\text {off }}$ and $\mathrm{G} 2$ arrest require cAMP-dependent Abl1 activation.

In summary, the model in Figure 8 describes extensive TORand cAMP/PKA-signaling integration that accounts for why i) germinating $\Delta a b l 1$ spores form appressoria after Rap but not cAMP treatment, ii) germinating $\Delta$ macl spores form appressoria after cAMP but not Rap treatment, and iii) germinating WT spores form appressoria on hydrophilic surfaces after either Rap or cAMP treatment.

\section{Conclusion.}

We conclude that integrated TOR and cAMP/PKA signaling mediates germ tube morphogenetic decision making under static and dynamic environmental conditions. However, unanswered questions remain. Why are nutrient-sensing and surface-sensing pathways wired together so extensively? Why do exogenous glucose treatments and hydrophilic surfaces both promote mitosis and germ tube growth, even if the latter condition is nutrient-free? Why are additional rounds of germ tube mitosis appressorially competent if G2 later becomes arrested? We propose that the model illustrated in Figure 8 not only ensures robust appressorial development under favorable conditions but, by allowing i) hydrophilic surfaces to activate TOR nutrient-signaling and ii) glucose-rich surfaces to inactivate cAMP/PKA surface-sensing, this integrated network also facilitates cell cycle-driven germ tube growth under any unfavorable condition. Considering that appressorial morphogenesis following extended germ tube growth and multiple mitotic rounds is permissible, we suggest that these observations indicate a hitherto unrecognized and potentially exploitable growth-mediated escape strategy, enabling spores germinating under noninductive conditions to reach favorable surfaces, form an appressorium, and cause devastating plant disease.

In yeast, the regulation of cell growth and stress responses by TOR and cAMP/PKA is complex (MacGilvray et al. 2018). Our work here, which connects integrated TOR and cAMP/PKA signaling to cellular growth and development, may serve as a gateway for deeper understanding of these fundamental and ancient signaling pathways. Future work to further our knowledge of this intricate system might involve determining where and how G2 arrest alleviates cAMP/PKA-signaling inhibition, and how $\mathrm{TOR}_{\text {off }}$ regulates Mac1 function.

\section{MATERIALS AND METHODS}

Strains and maintenance.

Guy11 was the WT strain used in this study (MarroquinGuzman et al. 2017b). Mutants were derived from Guy11 unless otherwise stated. The strains used in this work are shown in Supplementary Table S2. For routine maintenance, strains were grown on complete media (CM). CM contains $1 \%$ (wt/vol) glucose, $0.2 \%(\mathrm{wt} / \mathrm{vol})$ peptone, $0.1 \%(\mathrm{wt} / \mathrm{vol})$ yeast extract, and $0.1 \%$ (wt/vol) casamino acids and has a $\mathrm{pH}$ adjusted to 7.5 with $\mathrm{NaOH}$. Colonies were grown at $26^{\circ} \mathrm{C}$ under $12-\mathrm{h}$ light/dark cycles.

\section{Targeted gene replacement.}

$M$. oryzae protoplasts were generated and transformed using previously described methods (Marroquin-Guzman et al. 2017a). The $\triangle a \operatorname{tg} 8$ single mutant strain was generated by replacing the entire coding region of ATG8 (MGG_01062) with the ILVI gene conferring sulfurnoyl urea resistance using the PCR-based split marker method (Wilson et al. 2010). Briefly, $1 \mathrm{~kb}$ each from the left flank (LF) and right flank (RF) of the ATG8 coding region were amplified using the primers ATG8LF5' and ATG8-LF3' and ATG8-RF5' and ATG8-RF3', respectively. The $5^{\prime}$ region of the $I L V I$ gene was amplified using the primer pair M13F:SU and SuSplit, and the $3^{\prime}$ region of the ILV1 gene was amplified using the primer pair M13R:UR and UrSplit. The ATG8 left flank amplicon and the $5^{\prime}$ region of the ILVI gene were fused by amplifying with ATG8-NesF and

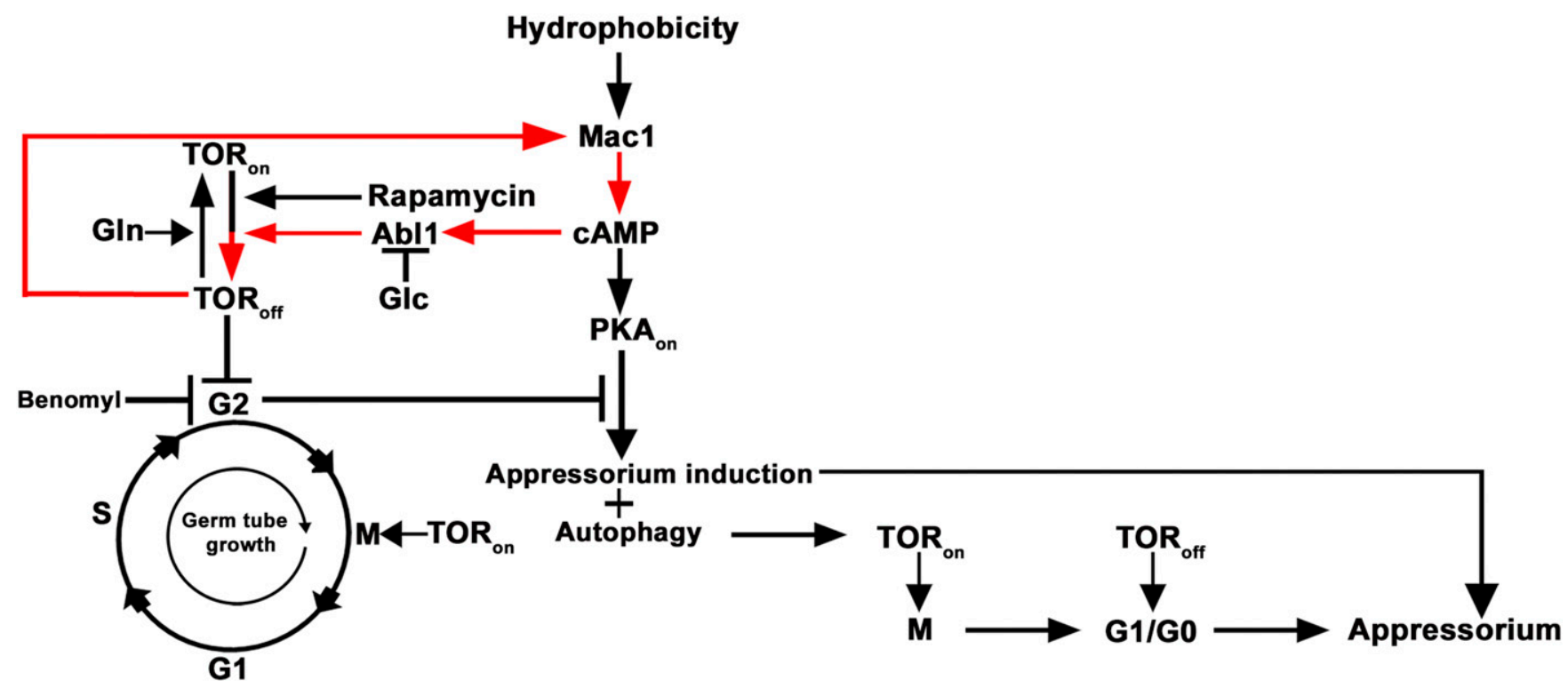

Fig. 8. Architecture of integrated TOR- and cAMP/PKA-signaling during spore germination. Integrated TOR- and cAMP/PKA-signaling via G2 underpins germ tube morphogenetic decision-making in response to static and changing environmental conditions. A feed-forward subnetwork reinforcing appressorium

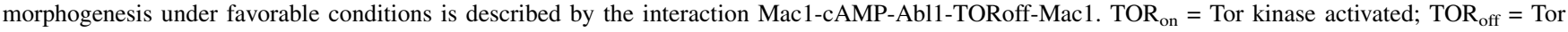
kinase inactivated; Glc = glucose; Gln = glutamine. 
SuSplit. The ATG8 right flank amplicon and the $3^{\prime}$ region of the ILVI gene were fused by amplifying with ATG8-NesR and UrSplit. The two resulting fragments, which overlap in the ILVI gene by approximately $300 \mathrm{bp}$, were transformed into protoplasts of the WT Guy11strain and were initially screened by BDCM-TOP media containing sulfurnoyl urea. Targeted gene deletion was confirmed by amplification of the flanking region of ATG8 with primers ATG8-LF5' and ATG8-RF3' . In a similar manner, the $\Delta a b l 1 \Delta c p k a$ double mutant was generated by replacing the entire coding region of $A B L 1$ (MGG_00987) in the $\Delta c p k a$ mutant strain with the $I L V I$ gene. The $\Delta m a c l$ single mutant was generated by replacing the entire coding region of MAC1 (MGG_09898) with the Bar gene conferring resistance to bialaphos. Primers sequences are shown in Supplementary Table S3.

\section{GFP:ATG8 fusion plasmid construction.}

The enhanced GFP (eGFP)-encoding gene was amplified from the pDL2 vector (Zhou et al. 2011) using the primes PDL2-eGFP-FW and eGFP-PDL2-RE. The ATG8 coding sequence (MGG_01062) was amplified from the M. oryzae genome DNA using the primers N-ATG8-C-eGFP-FW and C-ATG8-eGFP-RE. The resulting product carried 3'-eGFP sequences at the $5^{\prime}$ end. PCR fused the eGFP and ATG8 PCR products using the primers PDL2-GFP-FW and C-ATG8-eGFPRE. The fusion products were purified and transformed into Saccharomyces cerevisiae strain XK1-25 along with linearized pDL2, as described by following Zhou et al. (2011). The resulting plasmid was extracted from $S$. cerevisiae and transformed into Escherichia coli strain JM109 following the protocol provided with the kit (Promega). Following purification using the bacteria mini prep kit (Qiagen), $10 \mu \mathrm{g}$ of plasmid was transformed into WT Guy11 protoplasts. Positive transformants were selected by hygromycin resistance, screened by PCR, and confirmed by confocal microscopy.

\section{Spore germination and appressorium morphogenesis assays.}

Spores were harvested from 10- to 12-day-old colonies grown on CM, as described previously (Marroquin-Guzman et al. 2017b). Briefly, $5 \mathrm{ml}$ of $\mathrm{ddiH}_{2} \mathrm{O}$ was added to each plate, and spores were released by gentle scraping using a triangle spreader. Spore suspensions were filtered through two layers of sterile Miracloth into a 15-ml centrifuge tube. Distilled deionized water was added up to $15 \mathrm{ml}$. After centrifugation at $1,500 \times g$ for $10 \mathrm{~min}$, the water was decanted, and spore pellets were resuspended in $5 \mathrm{ml}$ of $\mathrm{ddiH}_{2} \mathrm{O}$ followed by centrifugation at $1,500 \times g$ for $10 \mathrm{~min}$. Spore pellets were resuspended in $1 \mathrm{ml}$ of $\mathrm{ddiH}_{2} \mathrm{O}$ and quantified using a hemocytometer. Spores suspensions were adjusted to give a final concentration of $5 \times 10^{4}$ spores per ml. Portions of the spore suspensions $(200$ or $100 \mu \mathrm{l})$ were used to inoculate inductive hydrophobic plastic coverslips (Fisherbrand) or noninductive three-welled glass slides (Fisherbrand). The inoculated slides were placed in a dark humidity chamber for $24 \mathrm{hpi}$, unless otherwise specified, followed by confocal microscopy. Appressoria formation rates were assessed by counting the number of appressoria formed from 50 spores per coverslip or slide, which was repeated with at least three technical replicates and three biological replicates per treatment, as described previously (Marroquin-Guzman et al. 2017b). Morphotypes were determined at $24 \mathrm{hpi}$ from 50 germinating spores per coverslip and repeated with six technical replicates and three biological replicates per treatment, as described previously (Marroquin-Guzman et al. 2017b). Nuclei number was determined from 100 spores per coverslip, using at least three technical replicates and three biological replicates per strain and treatment. WT and $\Delta a b l 1$ nuclei were visualized using WT H1:RFP and abl1 H1:RFP strains. $\Delta m a c 1$ nuclei were visualized using the SYTO green fluorescent nucleic acid stain (described below). Analysis of variance was performed with InfoStat software. All experiments were performed at $22^{\circ} \mathrm{C}$.

Exogenous treatments during appressorial morphogenesis.

All treatments were directly applied, at the indicated times, to spore suspensions previously inoculated onto hydrophobic or hydrophilic surfaces. Treatments included 100 to $200 \mathrm{nM}$ Rapamycin (Rap; LC Laboratories), $10 \mathrm{mM}$ monobutyryl cyclic AMP (cAMP, Sigma-Aldrich), 50 mM HU (Fisher Scientific), $30 \mu \mathrm{M}$ Ben (Fisher Scientific), 2 mM CHX (SigmaAldrich), $1 \%$ (wt/vol) glucose (Fisher Scientific), 0.02\% (wt/vol) Calcofluor white (Sigma Aldrich), and $100 \mathrm{mM}$ monodansylcadaverine crystallin (MDC) (Sigma-Aldrich). Staining by SYTO green (Thermo Fisher Scientific) was performed as described previously (Marroquin-Guzman et al. 2017b).

\section{Microscopy settings.}

Images were taken at the University of Nebraska-Lincoln using a Nikon A1 laser scanning confocal mounted on a Nikon 90i compound microscope (software version NIS Elements 4.13 Build914). A $561.5 \mathrm{~nm}$ laser was used to image transmitted light and td tomato fluorescence. The td tomato fluorescence was detected at 570 to $620 \mathrm{~nm}$. MDC and Calcofluor white fluorescence were detected at 425 to $475 \mathrm{~nm}$. SYTO green fluorescence was excited with a $488 \mathrm{~nm}$ filter and viewed with a 500 to $550 \mathrm{~nm}$ laser.

\section{ACKNOWLEDGMENTS}

We thank J. D. Wright and C. Elowsky, University of Nebraska-Lincoln, for technical assistance.

\section{LITERATURE CITED}

Choi, W., and Dean, R. A. 1997. The adenylate cyclase gene MAC1 of Magnaporthe grisea controls appressorium formation and other aspects of growth and development. Plant Cell 9:1973-1983.

Crespo, J. L., and Hall, M. N. 2002. Elucidating TOR signaling and rapamycin action: Lessons from Saccharomyces cerevisiae. Microbiol. Mol. Biol. Rev. 66:579-591.

Dagdas, Y. F., Yoshino, K., Dagdas, G., Ryder, L. S., Bielska, E., Steinberg, G., and Talbot, N. J. 2012. Septin-mediated plant cell invasion by the rice blast fungus, Magnaporthe oryzae. Science 336:1590-1595.

Di Pietro, A., and Talbot, N. J. 2017. Fungal pathogenesis: Combatting the oxidative burst. Nat. Microbiol. 2:17095.

Emmett, R. W., and Parbery, D. G. 1975. Appressoria. Annu. Rev. Phytopathol. 13:147-165.

Fernandez, J., Marroquin-Guzman, M., and Wilson, R. A. 2014. Evidence for a transketolase-mediated metabolic checkpoint governing biotrophic growth in rice cells by the blast fungus Magnaporthe oryzae. PLoS Pathog. 10:e1004354.

Fernandez, J., and Wilson, R. A. 2012. Why no feeding frenzy? Mechanisms of nutrient acquisition and utilization during infection by the rice blas fungus Magnaporthe oryzae. Mol. Plant-Microbe Interact 25:1286-1293.

Fisher, M. C., Henk, D. A., Briggs, C. J., Brownstein, J. S., Madoff, L. C., McCraw, S. L., and Gurr, S. J. 2012. Emerging fungal threats to animal, plant and ecosystem health. Nature 484:186-194.

Fleming, A., Noda, T., Yoshimori, T., and Rubinsztein, D. C. 2011 Chemical modulators of autophagy as biological probes and potential therapeutics. Nat. Chem. Biol. 7:9-17.

Hallett, J. E. H., Luo, X., and Capaldi, A. P. 2014. State transitions in the TORC1 signaling pathway and information processing in Saccharomyces cerevisiae. Genetics 198:773-786.

He, M., Kershaw, M. J., Soanes, D. M., Xia, Y., and Talbot, N. J. 2012 Infection-associated nuclear degeneration in the rice blast fungus Magnaporthe oryzae requires non-selective macro-autophagy. PLoS One 7:e33270.

Kershaw, M. J., and Talbot, N. J. 2009. Genome-wide functional analysis reveals that infection-associated fungal autophagy is necessary for rice blast disease. Proc. Natl. Acad. Sci. U.S.A. 106:15967-15972. 
Klionsky, D. J., Abdelmohsen, K., Abe, A., Abedin, M. J., Abeliovich, H., and Acevedo Arozena, A., et al. 2016. Guidelines for the use and interpretation of assays for monitoring autophagy (3rd edition). Autophagy 12:1-222.

Krick, R., Muehe, Y., Prick, T., Bremer, S., Schlotterhose, P., Eskelinen, E. L., Millen, J., Goldfarb, D. S., and Thumm, M. 2008. Piecemeal microautophagy of the nucleus requires the core macroautophagy genes. Mol. Biol. Cell 19:4492-4505.

Loewith, R., and Hall, M. N. 2011. Target of rapamycin (TOR) in nutrient signaling and growth control. Genetics 189:1177-1201.

MacGilvray, M. E., Shishkova, E., Chasman, D., Place, M., Gitter, A., Coon, J. J., and Gasch, A. P. 2018. Network inference reveals novel connections in pathways regulating growth and defense in the yeast salt response. PLoS Comput. Biol. 13:e1006088.

Marroquin-Guzman, M., Hartline, D., Wright, J. D., Elowsky, C., Bourret, T. J., and Wilson, R. A. 2017a. The Magnaporthe oryzae nitrooxidative stress response suppresses rice innate immunity during blast disease. Nat. Microbiol. 2:17054.

Marroquin-Guzman, M., Sun, G., and Wilson, R. A. 2017b. Glucose- $A B L 1$ TOR signaling modulates cell cycle tuning to control terminal appressorial cell differentiation. PLoS Genet. 13:e1006557.
Marroquin-Guzman, M., and Wilson, R. A. 2015. GATA-dependent glutaminolysis drives appressorium formation in Magnaporthe oryzae by suppressing TOR inhibition of cAMP/PKA signaling. PLoS Pathog. 11:e1004851.

Veneault-Fourrey, C., Barooah, M., Egan, M., Wakley, G., and Talbot, N. J. 2006. Autophagic fungal cell death is necessary for infection by the rice blast fungus. Science 312:580-583.

Wilson, R. A., Gibson, R. P., Quispe, C. F., Littlechild, J. A., and Talbot, N. J. 2010. An NADPH-dependent genetic switch regulates plant infection by the rice blast fungus. Proc. Natl. Acad. Sci. U.S.A. 107:21902-21907.

Wilson, R. A., and Talbot, N. J. 2009. Under pressure: Investigating the biology of plant infection by Magnaporthe oryzae. Nat. Rev. Microbiol. 7:185-195.

Xu, J. R., and Hamer, J. E. 1996. MAP kinase and cAMP signaling regulate infection structure formation and pathogenic growth in the rice blast fungus Magnaporthe grisea. Genes Dev. 10:2696-2706.

Xu, J.-R., Urban, M., Sweigard, J. A., and Hamer, J. E. 1997. The CPKA gene of Magnaporthe grisea is essential for appressorial penetration. Mol. Plant-Microbe Interact. 10:187-194.

Zhou, X., Li, G., and Xu, J. R. 2011. Efficient approaches for generating GFP fusion and epitope-tagging constructs in filamentous fungi. Methods Mol. Biol. 722:199-212. 